\title{
Construction of Fractional Power Series Solutions to Fractional Boussinesq Equations Using Residual Power Series Method
}

\author{
Fei Xu, ${ }^{1}$ Yixian Gao, ${ }^{1}$ Xue Yang, ${ }^{1}$ and He Zhang $^{2}$ \\ ${ }^{1}$ School of Mathematics and Statistics, Center for Mathematics and Interdisciplinary Sciences, \\ Northeast Normal University, Changchun, Jilin 130024, China \\ ${ }^{2}$ College of Mathematics, Jilin University, Changchun 130012, China
}

Correspondence should be addressed to Yixian Gao; gaoyx643@nenu.edu.cn

Received 19 January 2016; Accepted 11 April 2016

Academic Editor: Riccardo Caponetto

Copyright (C) 2016 Fei Xu et al. This is an open access article distributed under the Creative Commons Attribution License, which permits unrestricted use, distribution, and reproduction in any medium, provided the original work is properly cited.

\begin{abstract}
This paper is aimed at constructing fractional power series (FPS) solutions of time-space fractional Boussinesq equations using residual power series method (RPSM). Firstly we generalize the idea of RPSM to solve any-order time-space fractional differential equations in high-dimensional space with initial value problems in $\mathbb{R}^{n}$. Using RPSM, we can obtain FPS solutions of fourth-, sixth-, and $2 n$ th-order time-space fractional Boussinesq equations in $\mathbb{R}$ and fourth-order time-space fractional Boussinesq equations in $\mathbb{R}^{2}$ and $\mathbb{R}^{n}$. Finally, by numerical experiments, it is shown that RPSM is a simple, effective, and powerful method for seeking approximate analytic solutions of fractional differential equations.
\end{abstract}

\section{Introduction}

The study of nonlinear problems is of crucial importance not only in mathematics but also in physics, engineering, economic, and other disciplines, since most phenomena in our world are essentially nonlinear. But it is very difficult to establish mathematical models for nonlinear problem. In the field of engineering and science, we come across physical and natural phenomena which are represented by mathematical models, happening to differential equations. For example, simple harmonic motion, equation of motion, and deflection of a beam are represented by differential equations. Hence, the behaviour of differential equations is a necessity in such studies. A natural idea is to seek the exact solution, but it is often too difficult to succeed. So, as in [1], we attempt to construct approximate solution. Many assumptions have to be made artificially or unnecessarily in the integer-order differential system to make the practical problems solvable, leading to loss of most significant information. So it is necessary to construct new systems to solve these problems and fractional-order system is one of these. That is to say, fractional differential system is much well suited to physical problems compared to its differential partner since it makes less unnecessary or overrestricted assumption which may change the problem being solved, sometimes seriously [2].

The subject of fractional-order system dates back from the idea of derivatives of noninteger order which initially appeared in a letter from Leibnitz to L'Hospital in 1695 about the notation $d^{n} y / d x^{n}$. L'Hospital posed a question to Leibnitz: "what would the result be if $n=1 / 2$ ?" Leibnitz replied [3] "it follows that $d^{1 / 2} x$ will be equal to $x \sqrt{d x: x}$. This is an apparent paradox, from which, one day useful consequences will be drawn." From these words, fractional calculus was born. And so most authors on this topic will cite a particular date (September 30,1695) as the birthday of the so-called "fractional calculus" [4]. However, at that time, there are little specific models based on this kind of derivative, so the study of fractional order system attracts little attention.

Nowadays, more and more researchers pay massive attention to fractional calculus and they find that fractional systems can seize the missed information in the integral order systems. It is well known that the integer-order differential operators and the integer-order integral operators are local, but on the aspect as well, the fractional-order differential operators and the fractional-order integral operators are nonlocal operators. This means that the next state of a system 
depends not only upon its current state but also upon all of its historical states. In fact, this is the main reason why differential operators of fractional order provide an excellent instrument for description of memory and hereditary properties of various mathematical, physical, and engineering processes $[5,6]$. More details about the fractional differential theory can be found in [7-13].

Particularly, power series has played an important role in the study of elementary functions in the theory of calculus and it has been widely used in computational science for easily obtaining an approximation of functions. In physics, chemistry, and many other sciences, this power series expansion has allowed scientists to make an approximate study of many systems, neglecting higher-order terms around the equilibrium point. This is a fundamental tool to linearize a problem, which guarantees easy analysis [1, 6, 14-16].

The motivation of this paper is to study the time-space fractional Boussinesq equations. The motion of water waves plays an important role in oceanographic engineering and for most geographical areas, which are produced by environmental actions on beaches or on man-made fixed or floating structures. Natural wave trains are irregular in shape and they interact because the propagation process is nonlinear. Classical wave theory failed to describe the combined effect of these processes and they can be divided into two categories:

(1) a nonlinear description of monochromatic waves of a specific frequency or wave length;

(2) a linear description of irregular waves based on superposition of individual frequency components with random phases $[17,18]$.

So many mathematicians and physicists tried to contract new model to describe the process of wave propagation on the surface of water. In 1872, the French scientist Joseph Boussinesq (1842-1929) derived a model equation [18]

$$
\begin{aligned}
& \\
u_{t t}-u_{x x}+\mu u_{x x x x}=\eta\left(u^{2}\right)_{x x}, & \\
& -\infty<x<+\infty, t \geqslant 0,
\end{aligned}
$$

for the propagation of water waves from Euler's equations to describe the propagation process of long waves on the surface of water with small amplitude. This equation was formulated for analysis of long waves in shallow water. Later the general form of Boussinesq equation [17]

$$
u_{t t}-(f(u))_{x x}-u_{x x x x}=h(x, t),
$$

$$
-\infty<x<+\infty, t \geqslant 0
$$

was applied to problems not only in the study of the dynamics of thin inviscid layers with free surface but also in the study of nonlinear string, the shape-memory alloys, the propagation of waves in elastic rods, shallow wave waters (waves with long wavelength compared to the depth), and the continuum limit of lattice dynamics or coupled electrical circuits. More and more researchers pay attention to this equation and get a number of interesting results which were applied to many areas [17-20]. In recent years, with the development of fractional system rapidly, the classical Boussinesq equation evolves into the fractional Boussinesq equation, which is suitable for studying the water propagation through heterogeneous porous media [21-24].

This paper is concerned with the following initial value problem for time-space fractional Boussinesq equation in high-dimensional space:

$$
\begin{aligned}
D_{t}^{2 \alpha} u(x, t)= & \sum_{p=1}^{n_{1}} \beta_{p} D_{x_{p}}^{4 \sigma_{p}} u(x, t)+\sum_{q=1}^{n_{2}} \gamma_{q} D_{x_{q}}^{2 \tau_{q}} u(x, t) \\
& +\sum_{r=1}^{n} \theta_{r} D_{x_{r}}^{2 \lambda_{r}} u^{2}(x, t) \\
& -\sum_{r=1}^{n} 4 \theta_{r} u^{2}(x, t), \\
u(x, 0)= & a_{0}(x), \\
\left.D_{t}^{\alpha} u(x, t)\right|_{t=0}= & a_{1}(x),
\end{aligned}
$$

where $u(x, t)$ is a field function, $x=\left(x_{1}, x_{2}, \ldots, x_{n}\right) \in \mathbb{R}^{n}$, $n \in \mathbb{N}, t \in \mathbb{R}, 3 / 4<\sigma_{p} \leqslant 1, p=1,2, \ldots, n_{1} \leqslant n \in$ $\mathbb{N}, 1 / 2<\tau_{q} \leqslant 1, q=1,2, \ldots, n_{2} \leqslant n \in \mathbb{N}, 1 / 2<$ $\lambda_{r} \leqslant 1, r=1,2, \ldots, n, 1 / 2<\alpha \leqslant 1$, describing the order of the time-fractional derivative. The constants $\beta_{p}(p=$ $\left.1,2, \ldots, n_{1}\right)$ and $\gamma_{q}\left(q=1,2, \ldots, n_{2}\right)$ are the fourth-order and second-order coefficients of the space derivative, respectively, and the constants $\theta_{r}(r=1,2, \ldots, n)$ are the nonlinear coefficients. The symbol $D^{2 \alpha}$ means the Caputo fractional derivative of order $2 \alpha, a_{0}(x)$, and $a_{1}(x) \in C^{\infty}\left(\mathbb{R}^{n}\right)$. In [25], Jafari et al. considered the one-dimensional time-fractional Boussinesq equation; by finding the Lie point symmetries of the equation, they obtain the invariant solutions of the one-dimensional time-fractional Boussinesq equation. By the fractional subequation method, in [23], they obtained the solutions in terms of fractional hypergeometric functions, fractional triangle functions, and a rational function. Different from the solitary pattern solutions for nonlinear time-fractional dispersive partial differential equations in $\mathbb{R}$ obtained by Arqub et al. [15], in this paper we attempt to construct the more general approximate fractional power series solutions in $\mathbb{R}^{n}$ using RPSM. Furthermore, this method is valid for high-dimensional space and any-order time-space partial differential equations.

The organization of the rest of this paper is as follows: in Section 2, an introduction of some concepts on fractional calculus theory is presented. In Section 3, we introduce algorithm of RPSM for any-order time-space fractional differential equations with initial value problems. In Section 4, error analysis of RPSM is discussed simply. In Section 5, we apply RPSM to obtain fractional power series solutions of time-space fractional Boussinesq equations with initial value problems. The paper ends with some simple conclusions.

\section{Concepts on Fractional Calculus Theory}

There are several definitions of the fractional integration of order $\alpha \geq 0$, not necessarily equivalent to each other; 
see [9]. Riemann-Liouville and Caputo fractional definitions are the two most used from all the other definitions of fractional calculus which have been introduced recently [5, 6, 26-29].

Definition 1. The Mittag-Leffler function is defined as follows:

$$
E_{\alpha}(x):=\sum_{k=0}^{\infty} \frac{x^{k}}{\Gamma(\alpha k+1)}, \quad \alpha>0
$$

Definition 2. A real function $f(x), x>0$, is said to be in the space $C_{\mu}, \mu \in \mathbb{R}$, if there exists a real number $p>\mu$ such that $f(x)=x^{p} f_{1}(x)$, where $f_{1}(x) \in C[0,+\infty)$, and it is said to be in the space $C_{\mu}^{n}$ if $f^{(n)}(x) \in C_{\mu}, n \in \mathbb{N}$.

Definition 3. The Riemann-Liouville fractional integral operator of order $\alpha \geqslant 0$ of a function $f \in C_{\mu}, \mu \geqslant-1$, is defined as follows:

$$
\begin{aligned}
& J_{s}^{\alpha} f(x) \\
& := \begin{cases}\frac{1}{\Gamma(\alpha)} \int_{s}^{x}(x-t)^{\alpha-1} f(t) d t, & x>t>s \geqslant 0, \alpha>0, \\
f(x), & \alpha=0 .\end{cases}
\end{aligned}
$$

Property 1. Properties of the operator $J_{s}^{\alpha}$ : for $f \in C_{\mu}, \mu \geqslant-1$, $\alpha, \beta, C \in \mathbb{R}$, and $\gamma \geqslant-1$.

Property 1.1. Consider $J_{s}^{\alpha} J_{s}^{\beta} f(x)=J_{s}^{\alpha+\beta} f(x)=J_{s}^{\beta} J_{s}^{\alpha} f(x)$.

Property 1.2. Consider $J_{s}^{\alpha} C=(C / \Gamma(1+\alpha))(x-s)^{\alpha}$.

Property 1.3. Consider $J_{s}^{\alpha}(x-s)^{\gamma}=(\Gamma(\gamma+1) / \Gamma(\gamma+1+\alpha))(x-$ $s)^{\gamma+\alpha}$.

Definition 4. The Caputo fractional derivative of order $\alpha>0$ of $f \in C_{-1}^{n}, n \in \mathbb{N}$, is defined as follows:

$$
\begin{aligned}
& D_{s}^{\alpha} f(x) \\
& \quad:= \begin{cases}J_{s}^{n-\alpha} f^{(n)}(x), & x>s \geqslant 0, n-1<\alpha<n, \\
\frac{d^{n} f(x)}{d x^{n}}, & \alpha=n \in \mathbb{N} .\end{cases}
\end{aligned}
$$

Property 2. Properties of the operator $D_{s}^{\alpha}$ : for $\gamma>-1, x>s \geqslant$ 0 and $C \in \mathbb{R}$.

Property 2.1. Consider $D_{s}^{\alpha} D_{s}^{\beta} f(x)=D_{s}^{\alpha+\beta} f(x)=$ $D_{s}^{\beta} D_{s}^{\alpha} f(x)$.

Property 2.2. Consider $D_{s}^{\alpha} C=0$.

Property 2.3. Consider $D_{s}^{\alpha}(x-s)^{\gamma}=(\Gamma(\gamma+1) / \Gamma(\gamma+1-\alpha))(x-$ $s)^{\gamma-\alpha}$.
Definition 5. For $n$ to be the smallest integer that exceeds $\alpha$, the Caputo time-fractional derivative operator of order $\alpha$ of $u(x, t)$ is defined as follows:

$$
\begin{aligned}
& D_{t}^{\alpha} u(x, t) \\
& := \begin{cases}\frac{1}{\Gamma(n-\alpha)} \int_{0}^{t}(t-\tau)^{n-\alpha-1} \frac{\partial^{n} u(x, \tau)}{\partial \tau^{n}} d \tau, & n-1<\alpha<n, \\
\frac{\partial^{n} u(x, t)}{\partial t^{n}}, & \alpha=n \in \mathbb{N} .\end{cases}
\end{aligned}
$$

And the space fractional derivative of order $\beta$ is defined as follows:

$$
\begin{aligned}
& D_{x}^{\beta} u(x, t) \\
& := \begin{cases}\frac{1}{\Gamma(n-\beta)} \int_{0}^{x}(x-\tau)^{n-\beta-1} \frac{\partial^{n} u(\tau, t)}{\partial \tau^{n}} d \tau, & n-1<\beta<n, \\
\frac{\partial^{n} u(x, t)}{\partial x^{n}}, & \beta=n \in \mathbb{N} .\end{cases}
\end{aligned}
$$

Definition 6. A power series representation of the form

$$
\sum_{n=0}^{\infty} c_{n}\left(t-t_{0}\right)^{n \alpha}:=c_{0}+c_{1}\left(t-t_{0}\right)^{\alpha}+c_{2}\left(t-t_{0}\right)^{2 \alpha}+\cdots
$$

where $m-1<\alpha<m$ and $t \geqslant t_{0}$, is called a fractional power series about $t_{0}$, where $t$ is a variable and $c_{n}$ are the coefficients of the series.

Theorem 7 (see $[5,6])$. Suppose that $f$ has a FPS representation at $t_{0}$ of the form

$$
\begin{aligned}
& f(t)=\sum_{n=0}^{\infty} c_{n}\left(t-t_{0}\right)^{n \alpha}, \\
& 0 \leqslant m-1<\alpha \leqslant m, t_{0} \leqslant t<t_{0}+R,
\end{aligned}
$$

and $R$ is the radius of convergence of the FPS. If $f(t) \in C\left[t_{0}, t_{0}+\right.$ $R)$ and $D_{t}^{n \alpha} f(t) \in C\left(t_{0}, t_{0}+R\right)$ for $n=0,1,2, \ldots$, then the coefficients $c_{n}$ will take the form of

$$
c_{n}=\frac{\left.D_{t}^{n \alpha} f(t)\right|_{t=t_{0}}}{\Gamma(n \alpha+1)}
$$

where $D_{t}^{n \alpha}=D_{t}^{\alpha} \cdot D_{t}^{\alpha} \cdots \cdots D_{t}^{\alpha}($ n-times $)$.

Remark 8. If $f(t)=u(x, t)$, then we have

$$
\begin{aligned}
u(x, t) & =\sum_{n=0}^{\infty} f_{n}(x)\left(t-t_{0}\right)^{n \alpha}, \\
0 & \leqslant m-1<\alpha \leqslant m, x \in I \subset \mathbb{R}^{n}, t_{0} \leqslant t<t_{0}+R,
\end{aligned}
$$

and $R$ is the radius of convergence of the FPS. If $D_{t}^{n \alpha} \in C(I \times$ $\left.\left(t_{0}, t_{0}+R\right)\right), n=0,1,2, \ldots$, then the coefficients are $f_{n}(x)=$ $\left.D_{t}^{n \alpha} u(x, t)\right|_{t=t_{0}} / \Gamma(n \alpha+1)$. 


\section{Algorithm of RPSM}

In [14], Abu Arqub et al. give RPSM to obtain power series solution of higher-order ordinary differential equation with initial conditions in $\mathbb{R}$ in the integer system. In [5], El-Ajou et al. give the iterative progress of RPSM for time-fractional $\mathrm{KdV}$-Burgers equation in $\mathbb{R}$. In this section, we give the more general RPSM to find out fractional power series solution for any-order time-space fractional differential equations with initial conditions in $\mathbb{R}^{n}$.

Let us consider the higher-order time-space fractional differential equation as the following form:

$$
\begin{aligned}
D_{t}^{m \alpha} u(x, t)+G(x, t) & =0 \\
u(x, 0) & =a_{0}(x) \\
\left.D_{t}^{\alpha} u(x, t)\right|_{t=0} & =a_{1}(x), \ldots,\left.D_{t}^{(m-1) \alpha} u(x, t)\right|_{t=0} \\
& =a_{m-1}(x)
\end{aligned}
$$

where $x=\left(x_{1}, x_{2}, \ldots, x_{n}\right) \in I \subseteq \mathbb{R}^{n}, n \in \mathbb{N}, t \in \mathbb{R}$, and

$$
\begin{aligned}
& G(x, t)=F\left(u, D_{t}^{\alpha} u, D_{t}^{2 \alpha} u, \ldots, D_{t}^{(m-1) \alpha} u, D_{x_{1}}^{\beta_{11}} u, D_{x_{2}}^{\beta_{12}} u\right. \\
& \ldots, D_{x_{n}}^{\beta_{1 n}} u, D_{x_{1}}^{\beta_{21}} u, D_{x_{2}}^{\beta_{22}} u, \ldots, D_{x_{n}}^{\beta_{2 n}} u, \ldots, D_{x_{1}}^{\beta_{l 1}} u, D_{x_{2}}^{\beta_{l 2}} u, \ldots \\
& \left.D_{x_{n}}^{\beta_{l n}} u\right)
\end{aligned}
$$

$u(x, \cdot)$ and $G(x, \cdot)$ are analytic functions about $t,(m-1) / m<$ $\alpha \leqslant 1, m \in \mathbb{N}, p-1<\beta_{p j} \leqslant p(p=1,2, \ldots, l ; j=1,2, \ldots, n)$, and $a_{q}(x) \in C^{\infty}\left(\mathbb{R}^{n}\right), q=0,1,2, \ldots, m-1$.

Since $u(x, t)$ is analytic about $t$, the solution of the system can be written in the form of

$$
u(x, t)=\sum_{n=0}^{\infty} u_{n}(x, t)
$$

where $u_{n}(x, t)$ are terms of approximations and given as

$$
\begin{aligned}
& u_{n}(x, t)=C_{n}(x) t^{n \alpha}, \\
& x \in I \subset \mathbb{R}^{n},|t|<R, n=0,1,2, \ldots,
\end{aligned}
$$

where $R$ is the radius of convergence of above series. Obviously, when $i=0,1,2, \ldots, m-1$, since the terms $D_{t}^{i \alpha} u(x, t)$ satisfy the initial condition, we can get

$$
\begin{gathered}
a_{i}(x)=\left.D_{t}^{i \alpha} u(x, t)\right|_{t=0}=C_{i}(x) \Gamma(i \alpha+1) \Longrightarrow \\
u_{i}(x, t)=C_{i}(x) t^{i \alpha}=\frac{\left.D_{t}^{i \alpha} u(x, t)\right|_{t=0}}{\Gamma(i \alpha+1)} t^{i \alpha} \\
=\frac{a_{i}(x)}{\Gamma(i \alpha+1)} t^{i \alpha},
\end{gathered}
$$

$$
i=0,1, \ldots, m-1 \text {. }
$$

So we have the initial guess approximation of $u(x, t)$ in the following form:

$$
\begin{aligned}
u^{\text {initial }}(x, t):= & u_{0}(x, t)+u_{1}(x, t)+\cdots+u_{m-1}(x, t) \\
= & a_{0}(x)+\frac{a_{1}(x)}{\Gamma(\alpha+1)} t^{\alpha}+\cdots \\
& +\frac{a_{m-1}(x)}{\Gamma((m-1) \alpha+1)} t^{(m-1) \alpha} .
\end{aligned}
$$

On the other aspect as well, if we choose $u^{\text {initial }}(x, t)$ as initial guess approximation $u_{i}(x, t)$ for $i=m, m+1, m+2, \ldots$, the approximate of the solution of $u(x, t)$ of (13) by the $k$ th truncated series is

$$
\begin{aligned}
u^{k}(x, t):=u^{\text {initial }}(x, t)+\sum_{i=m}^{k} C_{i}(x) t^{i \alpha}, \\
\quad k=m, m+1, m+2, \ldots .
\end{aligned}
$$

Before applying RPS method for solving (13), we give some notes:

$$
\operatorname{Res}(x, t):=D_{t}^{m \alpha} u(x, t)+G(x, t) .
$$

Substituting the $k$ th truncated series $u^{k}(x, t)$ into (13), we can obtain the following definition for $k$ th residual function:

$$
\operatorname{Res}^{k}(x, t):=D_{t}^{m \alpha} u^{k}(x, t)+G^{k}(x, t),
$$

where

$$
\begin{aligned}
& G^{k}(x, t)=F\left(u^{k}, D_{t}^{\alpha} u^{k}, D_{t}^{2 \alpha} u^{k}, \ldots, D_{t}^{(m-1) \alpha} u^{k}, D_{x_{1}}^{\beta_{11}} u^{k}\right. \\
& D_{x_{2}}^{\beta_{12}} u^{k}, \ldots, D_{x_{n}}^{\beta_{1 n}} u^{k}, D_{x_{1}}^{\beta_{21}} u^{k}, D_{x_{2}}^{\beta_{22}} u^{k}, \ldots, D_{x_{n}}^{\beta_{2 n}} u^{k}, \ldots \\
& \left.D_{x_{1}}^{\beta_{l 1}} u^{k}, D_{x_{2}}^{\beta_{l 2}} u^{k}, \ldots, D_{x_{n}}^{\beta_{l n}} u^{k}\right) .
\end{aligned}
$$

Then we have the following facts:

(1) $\lim _{k \rightarrow \infty} u^{k}(x, t)=u(x, t)$;

(2) $\operatorname{Res}(x, t)=0$;

(3) $\operatorname{Res}^{\infty}(x, t)=\lim _{k \rightarrow+\infty} \operatorname{Res}^{k}(x, t)=\operatorname{Res}(x, t)=0, x \in$ $I \subseteq \mathbb{R}^{n},|t|<R$.

These show that the residual function $\operatorname{Res}^{\infty}(x, t)$ is infinitely many times differentiable at $t=0$. On the other hand, we can show that

$$
\begin{aligned}
0 & =\left.D_{t}^{(k-m) \alpha} \operatorname{Res}^{\infty}(x, t)\right|_{t=0} \\
& =C_{k}(x) \Gamma(k \alpha+1)+\left.D_{t}^{(k-m) \alpha} G^{k}(x, t)\right|_{t=0} \Longrightarrow \\
C_{k}(x) & =-\frac{\left.D_{t}^{(k-m) \alpha} G^{k}(x, t)\right|_{t=0}}{\Gamma(k \alpha+1)}
\end{aligned}
$$


Since $-\left.D_{t}^{(k-m) \alpha} G^{k}(x, t)\right|_{t=0}$ is not dependent on $t$, denoting it by $f_{k}(x)$, then (23) can be written as

$$
\begin{aligned}
C_{k}(x) & =\frac{f_{k}(x)}{\Gamma(k \alpha+1)}, \\
f_{k}(x) & =-\left.D_{t}^{(k-m) \alpha} G^{k}(x, t)\right|_{t=0}, \\
k & =m, m+1, m+2, \ldots .
\end{aligned}
$$

In fact, the relation of (24) is a fundamental rule in RPSM and its applications. So the FPS solution of (13) is

$$
\begin{aligned}
u(x, t) & =u^{\text {initial }}(x, t)+\sum_{i=m}^{\infty} C_{i}(x) t^{i \alpha} \\
& =\sum_{i=0}^{m-1} \frac{a_{i}(x)}{\Gamma(i \alpha+1)} t^{i \alpha}+\sum_{i=m}^{\infty} \frac{f_{i}(x)}{\Gamma(i \alpha+1)} t^{i \alpha}
\end{aligned}
$$

where $a_{i}(x)(i=0,1,2, \ldots, m-1)$ are given and $f_{i}(x)(i=$ $m, m+1, m+2, \ldots)$ have been constructed by RPSM in the form (24).

The algorithm of RPSM is considered a direct and simple approach because it depends on the recursive differentiation of time-fractional derivative and the use of given initial conditions to calculate coefficients of the multiple FPS solution using minimal calculations. The RPS does not require linearization, perturbation, or discretization of the variables; is not affected by computational round-off errors; and does not require large computer memory and extensive time.

\section{Error Analysis of RPSM}

It will be convenient to have a notation for the error in the approximation of (13):

$$
\begin{aligned}
u(x, t) & \approx u^{k}(x, t), \quad(k=0,1,2, \ldots), \\
\operatorname{Rem}^{k}(x, t) & =u(x, t)-u^{k}(x, t),
\end{aligned}
$$

where $u(x, t)$ denotes the exact solution of (13) and $u^{k}(x, t)$ denotes $k$ th approximate solution of (13) obtained by RPSM and $\operatorname{Rem}^{k}(x, t)$ denotes the difference between $u(x, t)$ and $u^{k}(x, t)$. The functions $\operatorname{Rem}^{k}(x, t)$ are called the $k$ th remainder for the approximation of $u(x, t)$. In fact, it often happens that the remainder $\operatorname{Rem}^{k}(x, t)$ become smaller and smaller, approaching zero, as $k$ tends to infinity.

To show the accuracy and efficiency of RPSM, we give two types of error:

$$
\operatorname{Exa}^{k}(x, t):=\left|\operatorname{Rem}^{k}(x, t)\right|=\left|u(x, t)-u^{k}(x, t)\right|,
$$

where $\operatorname{Exa}^{k}(x, t)$ denotes the absolute error;

$$
\operatorname{Rel}^{k}(x, t):=\frac{\left|\operatorname{Rem}^{k}(x, t)\right|}{|u(x, t)|}=\frac{\left|u(x, t)-u^{k}(x, t)\right|}{|u(x, t)|}
$$

where $\operatorname{Rel}^{k}(x, t)$ denotes the relative error.
RPSM provides an approximate analytical solution in terms of an infinite FPS and it is necessary to give the errors of the approximate solution. In the next section, we apply RPSM to time-space fractional Boussinesq equations and take fourth-order time-space fractional Boussinesq equations as an example to illustrate the absolute error and relative error of the approximate solution.

\section{Application of RPSM to Time-Fractional Boussinesq Equations}

In this section, the procedure of constructing approximate analytical solutions to time-space fractional Boussinesq equations using RPSM is presented.

5.1. Fourth-Order Time-Space Fractional Boussinesq Equations in $\mathbb{R}$. Consider the fourth-order time-space fractional Boussinesq equation with one-dimensional space variable:

$$
\begin{aligned}
D_{t}^{2 \alpha} u(x, t)= & \beta D_{x}^{4 \sigma} u(x, t)+\gamma D_{x}^{2 \tau} u(x, t) \\
& +\theta D_{x}^{2 \lambda} u^{2}(x, t)-4 \theta u^{2}(x, t), \\
u(x, 0)= & a_{0}(x), \\
\left.D_{t}^{\alpha} u(x, t)\right|_{t=0}= & a_{1}(x),
\end{aligned}
$$

where $u(x, t)$ is a field function, $(x, t) \in \mathbb{R} \times \mathbb{R}, 1 / 2<\alpha \leq 1$, $3 / 4<\sigma \leqslant 1,1 / 2<\tau \leqslant 1,1 / 2<\lambda \leqslant 1, \beta, \gamma$, and $\theta$ are constant coefficients, and $a_{0}(x)$ and $a_{1}(x) \in C^{\infty}(\mathbb{R})$.

According to (25), the solution of (29) can be written in the following form:

$$
\begin{aligned}
u(x, t) & =\sum_{n=0}^{\infty} u_{n}(x, t), \\
u_{n}(x, t) & =C_{n}(x) t^{n \alpha}=\frac{f_{n}(x)}{\Gamma(n \alpha+1)} t^{n \alpha},
\end{aligned}
$$

$$
n=0,1,2, \ldots
$$

Apparently, according to (17), it yields

$$
\begin{aligned}
& f_{0}(x)=a_{0}(x), \\
& f_{1}(x)=a_{1}(x) .
\end{aligned}
$$

By (18)-(22), we can show that

$$
\begin{aligned}
u^{\text {initial }}(x, t) & =a_{0}(x)+\frac{a_{1}(x)}{\Gamma(\alpha+1)} t^{\alpha}, \\
u^{k}(x, t) & =u^{\text {initial }}(x, t)+\sum_{i=2}^{k} \frac{f_{i}(x)}{\Gamma(i \alpha+1)} t^{i \alpha}, \\
k & =2,3, \ldots,
\end{aligned}
$$

$\operatorname{Res}(x, t)=D_{t}^{2 \alpha} u(x, t)+G(x, t)$, 
where $G(x, t)=-\beta D_{x}^{4 \sigma} u(x, t)-\gamma D_{x}^{2 \tau} u(x, t)-\theta D_{x}^{2 \lambda} u^{2}(x, t)+$ $4 \theta u^{2}(x, t)$,

$$
\begin{array}{rl}
\operatorname{Res}^{k}(x, t)= & D_{t}^{2 \alpha} u^{k}(x, t)+G^{k}(x, t), \\
G^{k}(x, t)= & -\beta D_{x}^{4 \sigma} u^{k}(x, t)-\gamma D_{x}^{2 \tau} u^{k}(x, t) \\
& -\theta D_{x}^{2 \lambda}\left(u^{k}(x, t)\right)^{2}+4 \theta\left(u^{k}(x, t)\right)^{2}, \\
k & k 2,3, \ldots .
\end{array}
$$

According to (23) and (24), we can obtain

$$
\begin{aligned}
f_{i}(x) & =-\left.D_{t}^{(i-2) \alpha} G^{i}(x, t)\right|_{t=0} \\
& =\left.\beta D_{t}^{(i-2) \alpha} D_{x}^{4 \sigma} u^{i}(x, t)\right|_{t=0} \\
& +\left.\gamma D_{t}^{(i-2) \alpha} D_{x}^{2 \tau} u^{i}(x, t)\right|_{t=0} \\
& +\left.\theta D_{t}^{(i-2) \alpha} D_{x}^{2 \lambda}\left(u^{i}(x, t)\right)^{2}\right|_{t=0} \\
& -\left.4 \theta D_{t}^{(i-2) \alpha}\left(u^{i}(x, t)\right)^{2}\right|_{t=0}=\beta D_{x}^{4 \sigma} a_{i-2}(x) \\
& +\gamma D_{x}^{2 \tau} a_{i-2}(x)+\theta D_{x}^{2 \lambda} \Gamma((i-2) \alpha+1) \\
& \cdot \sum_{j=0}^{i-2} \frac{a_{j}(x) a_{i-2-j}(x)}{\Gamma(j \alpha+1) \Gamma((i-2-j) \alpha+1)} \\
& -4 \theta \Gamma((i-2) \alpha+1) \\
& \cdot \sum_{j=0}^{i-2} \frac{a_{j}(x) a_{i-2-j}(x)}{\Gamma(j \alpha+1) \Gamma((i-2-j) \alpha+1)} \triangleq a_{i}(x), \\
& i=2,3,4, \ldots
\end{aligned}
$$

So the $k$ th approximate solution of (29) is

$$
u^{k}(x, t)=\sum_{i=0}^{k} \frac{a_{i}(x)}{\Gamma(i \alpha)+1} t^{i \alpha}, \quad k=0,1,2,3, \ldots,
$$

where $a_{i}(x)(i=0,1,2,3, \ldots)$ can be found from (29) and (34).

Particularly, when $m=2, \beta=1, \gamma=1, \theta=1, \sigma=1$, $\tau=1, \lambda=1, a_{0}(x)=e^{x}$, and $a_{1}(x)=\sqrt{2} e^{x}$, the exact solution of (29) is

$$
u(x, t)=e^{x} E_{\alpha}\left(\sqrt{2} t^{\alpha}\right) .
$$

Under these special conditions, according to RPSM, that is (31)-(34), we have

$$
a_{i}(x)=(\sqrt{2})^{i} e^{x}, \quad i=0,1,2,3, \ldots .
$$

And the $k$ th approximate solution of (29) is

$$
\begin{array}{r}
u^{k}(x, t)=e^{x} \sum_{i=0}^{k} \frac{a_{i}(x)}{\Gamma(i \alpha+1)} t^{i \alpha}=e^{x} \sum_{i=0}^{k} \frac{(\sqrt{2})^{i}}{\Gamma(i \alpha+1)} t^{i^{\alpha}}, \\
k=0,1,2,3, \ldots
\end{array}
$$

It is easy to see that

$$
\begin{aligned}
u(x, t) & =\lim _{k \rightarrow \infty} u^{k}(x, t)=\lim _{k \rightarrow \infty} e^{x} \sum_{i=0}^{k} \frac{(\sqrt{2})^{i}}{\Gamma(i \alpha+1)} t^{i \alpha} \\
& =e^{x} \sum_{n=0}^{\infty} \frac{\left(\sqrt{2} t^{\alpha}\right)^{n}}{\Gamma(n \alpha+1)}=e^{x} E_{\alpha}\left(\sqrt{2} t^{\alpha}\right) .
\end{aligned}
$$

So the FPS solution of (29) is

$$
u(x, t)=e^{x} E_{\alpha}\left(\sqrt{2} t^{\alpha}\right),
$$

which is equal to the exact solutions (36).

Since we choose the special constant coefficients and give the initial value $a_{0}(x)=e^{x}$ and $a_{1}(x)=\sqrt{2} e^{x}$, so the exact solution in (36) is easily obtained. However, the initial value in application may be "complex" which leads to difficulty to obtain the exact solution and even we cannot obtain it, so it is necessary to construct the approximate solution by RPSM. Thus it is necessary to illustrate the the validity and reliability of RPSM in order to construct numerical solution for generalized initial value.

In the numerical experiment, we take $\alpha=1$; then $u(x, t)=e^{x+\sqrt{2} t}$. We plot the image of $u(x, t)$ and $u^{9}(x, t)$ with the Mathematica software (see Figures 1 and 2). It is obvious that $u^{k}(x, t)$ is closely approximate to $u(x, t)$ when $k=9$. In order to give precise comparison, we plot the intersection of $u(x, t)$ and $u^{k}(x, t)$ at $x=t$ when $k=3,5,7,9$ with MATLAB (see Figures $3,4,5$, and 6). It is clear that $u^{k}(x, t)$ (see the red curve) is very close to $u(x, t)$ (see the blue curve) when $k=9$, especially around the point $(0,0)$ which corresponds to the form of the fractional power series solution assumed in (15).

In the Appendix, from the comparison of Tables 1-4, if the variable $x$ is given, the absolute error $\operatorname{Exa}^{k}(x, t)$ and the relative error $\operatorname{Rel}^{k}(x, t)$ tend to 0 as $t$ tends to 0 . So the behaviour of the exact solution can be described by the approximate fractional power series solution around the point $(0,0)$.

Furthermore, if we want to know the behaviour of $u(x, t)$ around the point $\left(x_{0}, t_{0}\right) \in \mathbb{R} \times(-R, R)$, we can get the information by the transformation $\Gamma:(0,0) \mapsto$ $\left(x_{0}, t_{0}\right)$, namely, the expansion of the exact solution at point $\left(x_{0}, t_{0}\right)$. And this analysis indicates that RPSM is effective and powerful in approximating the exact solution.

5.2. Sixth-Order Time-Space Fractional Boussinesq Equations in $\mathbb{R}$. Let us consider the sixth-order time-space fractional Boussinesq equation with one-dimensional space variable:

$$
\begin{aligned}
D_{t}^{2 \alpha} u(x, t)= & \delta D_{x}^{6 \rho} u(x, t)+\beta D_{x}^{4 \sigma} u(x, t) \\
& +\gamma D_{x}^{2 \tau} u(x, t)+\theta D_{x}^{2 \lambda} u^{2}(x, t) \\
& -4 \theta u^{2}(x, t), \\
u(x, 0)= & a_{0}(x), \\
\left.D_{t}^{\alpha} u(x, t)\right|_{t=0}= & a_{1}(x),
\end{aligned}
$$




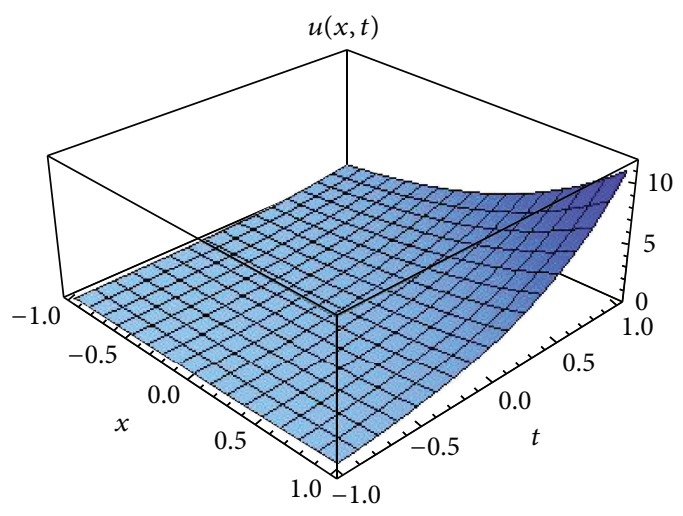

Figure 1: The image of $u(x, t)$.

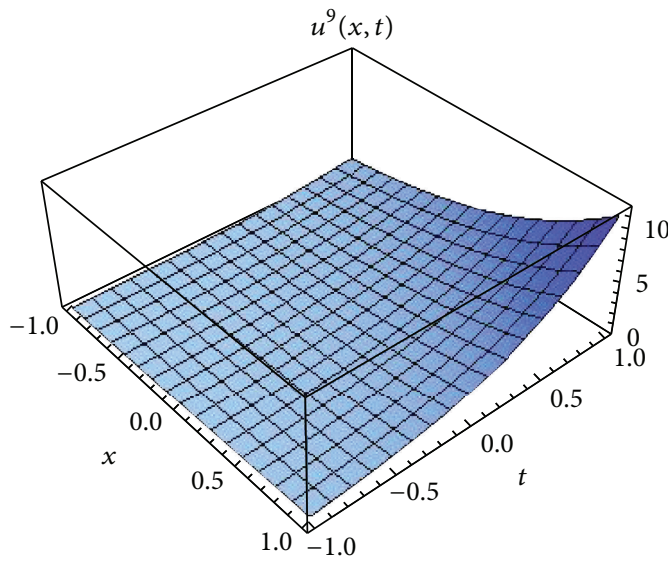

Figure 2: The image of $u^{9}(x, t)$.

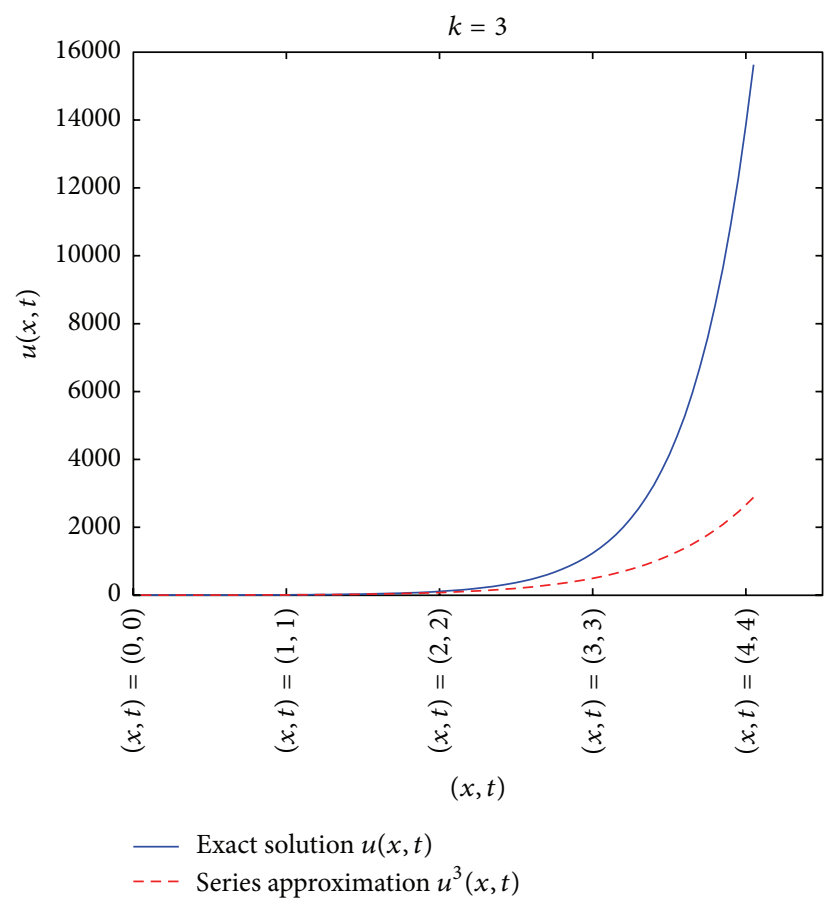

Figure 3: The image of $u(x, t)$ and $u^{3}(x, t)$.

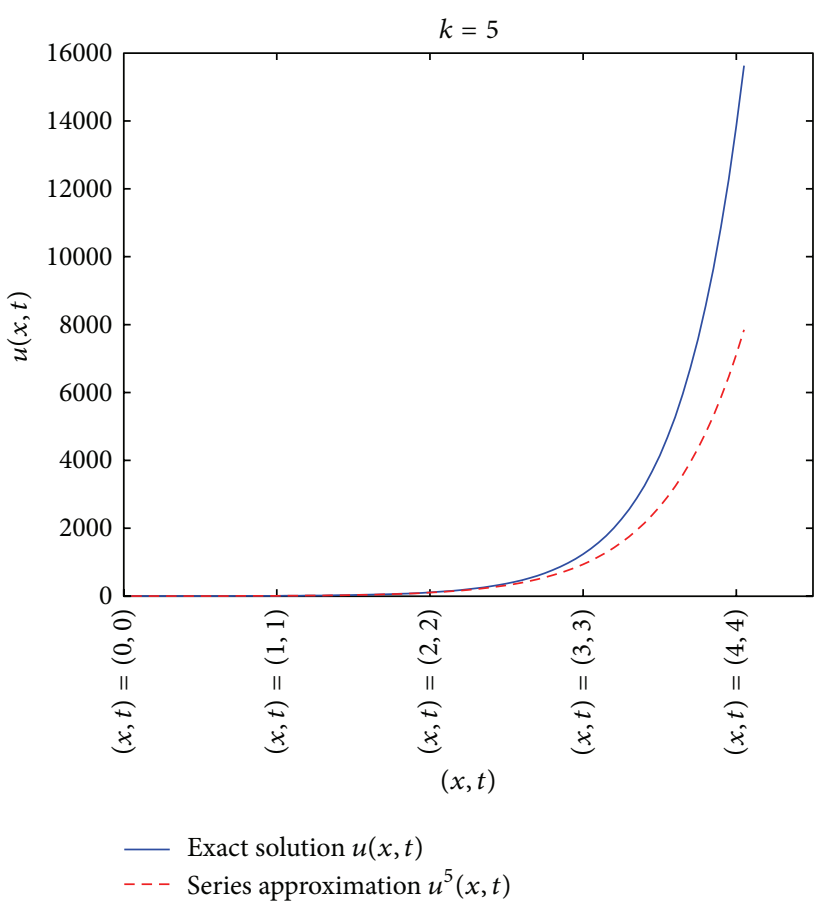

FIGURE 4: The image of $u(x, t)$ and $u^{5}(x, t)$.

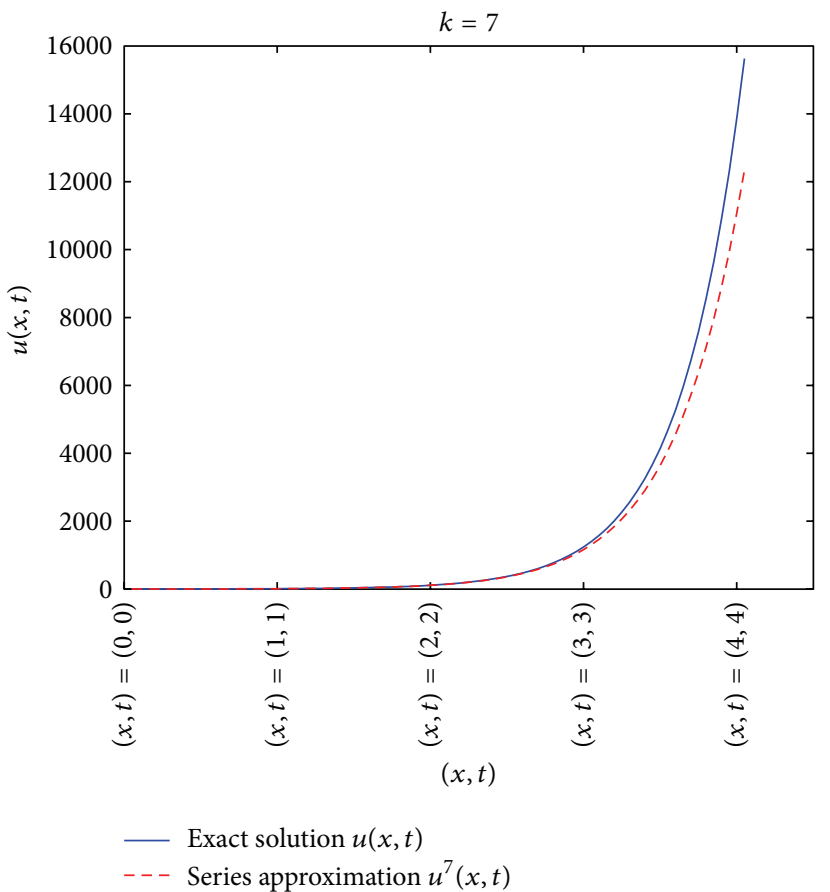

FIGURE 5: The image of $u(x, t)$ and $u^{7}(x, t)$.

where $u(x, t)$ is a field function, $(x, t) \in \mathbb{R} \times \mathbb{R}, 1 / 2<\alpha \leq 1$, $5 / 6<\rho \leq 1,3 / 4<\sigma \leq 1,1 / 2<\tau \leq 1,1 / 2<\lambda \leq 1, \delta, \beta, \gamma$, and $\theta$ are constants, and $a_{0}(x)$ and $a_{1}(x) \in C^{\infty}(\mathbb{R})$. 
TABLE 1: Absolute error and relative error of approximating the solution of (29) when $\alpha=\beta=\gamma=\theta=1$ and $a_{0}(x)=e^{x}$, $a_{1}(x)=\sqrt{2} e^{x}$ using RPSM at $x=5$.

\begin{tabular}{cccccc}
\hline$k$ & $t$ & $u(x, t)$ & $u^{k}(x, t)$ & $\mathrm{Exa}^{k}(x, t)$ & $\mathrm{Rel}^{k}(x, t)$ \\
\hline \multirow{4}{*}{3} & 1 & 610.4605 & 576.6769 & 33.7836 & 0.0553 \\
& 0.5 & 300.9990 & 299.2057 & 1.7932 & 0.0060 \\
& 0.25 & 211.3580 & 211.2541 & 0.1039 & 0.0005 \\
& 0.125 & 177.1110 & 177.1047 & 0.0063 & 0 \\
\hline \multirow{4}{*}{5} & 1 & 610.4605 & 608.4086 & 2.0518 & 0.0034 \\
& 0.5 & 300.9990 & 300.9703 & 0.0286 & 0.0001 \\
& 0.25 & 211.3580 & 211.3576 & 0.0004 & 0 \\
& 0.125 & 177.1110 & 177.1110 & 0 & 0 \\
\hline \multirow{4}{*}{7} & 1 & 610.4605 & 610.3908 & 0.0696 & 0.0001 \\
& 0.5 & 300.9990 & 300.9987 & 0.0002 & 0 \\
& 0.25 & 211.3580 & 211.3580 & 0 & 0 \\
& 0.125 & 177.1110 & 177.1110 & 0 & 0 \\
\hline \multirow{3}{*}{9} & 1 & 610.4605 & 610.4590 & 0.0015 & 0 \\
& 0.5 & 300.9990 & 300.9990 & 0 & 0 \\
& 0.25 & 211.3580 & 211.3580 & 0 & 0 \\
& 0.125 & 177.1110 & 177.1110 & 0 & 0 \\
\hline
\end{tabular}

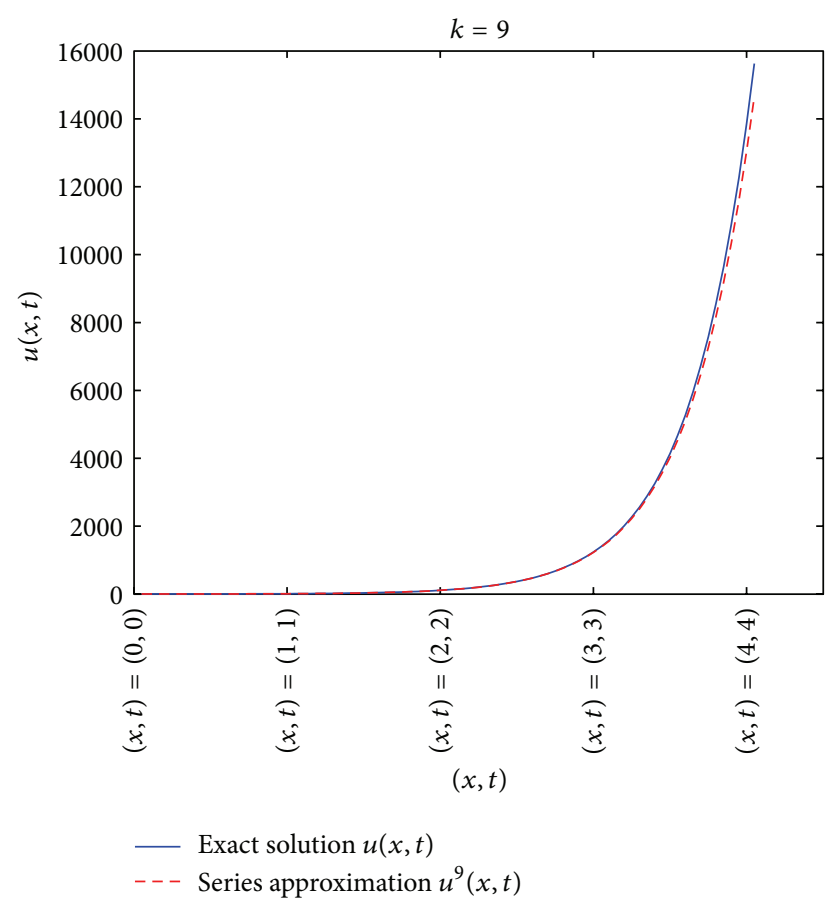

FIGURE 6: The image of $u(x, t)$ and $u^{9}(x, t)$.

According to (25), the solution of (41) can be written in the following form:

$$
\begin{array}{rl}
u(x, t) & =\sum_{n=0}^{\infty} u_{n}(x, t), \\
u_{n}(x, t) & =C_{n}(x) t^{n \alpha}=\frac{f_{n}(x)}{\Gamma(n \alpha+1)} t^{n \alpha}, \\
n & n=0,1,2, \ldots .
\end{array}
$$

TABLE 2: Absolute error and relative error of approximating the solution of (29) when $\alpha=\beta=\gamma=\theta=1$ and $a_{0}(x)=e^{x}$, $a_{1}(x)=\sqrt{2} e^{x}$ using RPSM at $x=2.5$.

\begin{tabular}{cccccc}
\hline$k$ & $t$ & $u(x, t)$ & $u^{k}(x, t)$ & $\mathrm{Exa}^{k}(x, t)$ & $\mathrm{Rel}^{k}(x, t)$ \\
\hline & 1 & 50.1096 & 47.3365 & 2.7731 & 0.0553 \\
3 & 0.5 & 24.7075 & 24.5603 & 0.1472 & 0.0060 \\
& 0.25 & 17.3493 & 17.3408 & 0.0085 & 0.0005 \\
& 0.125 & 14.5382 & 14.5376 & 0.0005 & 0 \\
\hline \multirow{4}{*}{5} & 1 & 50.1096 & 49.9412 & 0.1684 & 0.0034 \\
& 0.5 & 24.7075 & 24.7051 & 0.0023 & 0.0001 \\
& 0.25 & 17.3493 & 17.3493 & 0 & 0 \\
& 0.125 & 14.5382 & 14.5382 & 0 & 0 \\
\hline \multirow{3}{*}{7} & 1 & 50.1096 & 50.1039 & 0.0057 & 0.0001 \\
& 0.5 & 24.7075 & 24.7075 & 0 & 0 \\
& 0.25 & 17.3493 & 17.3493 & 0 & 0 \\
& 0.125 & 14.5382 & 14.5382 & 0 & 0 \\
\hline \multirow{3}{*}{9} & 1 & 50.1096 & 50.1095 & 0.0001 & 0 \\
& 0.5 & 24.7075 & 24.7075 & 0 & 0 \\
& 0.25 & 17.3493 & 17.3493 & 0 & 0 \\
& 0.125 & 14.5382 & 14.5382 & 0 & 0 \\
\hline
\end{tabular}

TABLE 3: Absolute error and relative error of approximating the solution of (29) when $\alpha=\beta=\gamma=\theta=1$ and $a_{0}(x)=e^{x}$, $a_{1}(x)=\sqrt{2} e^{x}$ using RPSM at $x=1.25$.

\begin{tabular}{cccccc}
\hline$k$ & $t$ & $u(x, t)$ & $u^{k}(x, t)$ & $\mathrm{Exa}^{k}(x, t)$ & $\mathrm{Rel}^{k}(x, t)$ \\
\hline \multirow{3}{*}{3} & 1 & 14.3567 & 13.5621 & 0.7945 & 0.0553 \\
& 0.5 & 7.0788 & 7.0366 & 0.0422 & 0.0060 \\
& 0.25 & 4.9707 & 4.9682 & 0.0024 & 0.0005 \\
& 0.125 & 4.1653 & 4.1651 & 0.0001 & 0 \\
\hline \multirow{4}{*}{5} & 1 & 14.3567 & 14.3084 & 0.0483 & 0.0034 \\
& 0.5 & 7.0788 & 7.0781 & 0.0007 & 0.0001 \\
& 0.25 & 4.9707 & 4.9707 & 0 & 0 \\
& 0.125 & 4.1653 & 4.1653 & 0 & 0 \\
\hline \multirow{4}{*}{7} & 1 & 14.3567 & 14.3550 & 0.0016 & 0.0001 \\
& 0.5 & 7.0788 & 7.0788 & 0 & 0 \\
& 0.25 & 4.9707 & 4.9707 & 0 & 0 \\
& 0.125 & 4.1653 & 4.1653 & 0 & 0 \\
\hline \multirow{4}{*}{9} & 1 & 14.3567 & 14.3566 & 0 & 0 \\
& 0.5 & 7.0788 & 7.0788 & 0 & 0 \\
& 0.25 & 4.9707 & 4.9707 & 0 & 0 \\
& 0.125 & 4.1653 & 4.1653 & 0 & 0 \\
\hline
\end{tabular}

Apparently, according to (17), we have

$$
\begin{aligned}
& f_{0}(x)=a_{0}(x), \\
& f_{1}(x)=a_{1}(x) .
\end{aligned}
$$


TABLE 4: Absolute error and relative error of approximating the solution of (29) when $\alpha=\beta=\gamma=\theta=1$ and $a_{0}(x)=e^{x}$, $a_{1}(x)=\sqrt{2} e^{x}$ using RPSM at $x=0.625$.

\begin{tabular}{cccccc}
\hline$k$ & $t$ & $u(x, t)$ & $u^{k}(x, t)$ & $\mathrm{Exa}^{k}(x, t)$ & $\mathrm{Rel}^{k}(x, t)$ \\
\hline \multirow{4}{*}{3} & 1 & 7.6846 & 7.2593 & 0.4253 & 0.0553 \\
& 0.5 & 3.7890 & 3.7664 & 0.0226 & 0.0060 \\
& 0.25 & 2.6606 & 2.6593 & 0.0013 & 0.0005 \\
& 0.125 & 2.2295 & 2.2294 & 0.0001 & 0 \\
\hline & 1 & 7.6846 & 7.6587 & 0.0258 & 0.0034 \\
5 & 0.5 & 3.7890 & 3.7887 & 0.0004 & 0.0001 \\
& 0.25 & 2.6606 & 2.6606 & 0 & 0 \\
& 0.125 & 2.2295 & 2.2295 & 0 & 0 \\
\hline & 1 & 7.6846 & 7.6837 & 0.0009 & 0.0001 \\
7 & 0.5 & 3.7890 & 3.7890 & 0 & 0 \\
& 0.25 & 2.6606 & 2.6606 & 0 & 0 \\
& 0.125 & 2.2295 & 2.2295 & 0 & 0 \\
\hline \multirow{4}{*}{9} & 1 & 7.6846 & 7.6845 & 0 & 0 \\
& 0.5 & 3.7890 & 3.7890 & 0 & 0 \\
& 0.25 & 2.6606 & 2.6606 & 0 & 0 \\
& 0.125 & 2.2295 & 2.2295 & 0 & 0 \\
\hline
\end{tabular}

Using (18)-(22), we can get

$$
\begin{aligned}
u^{\text {initial }}(x, t) & =a_{0}(x)+\frac{a_{1}(x)}{\Gamma(\alpha+1)} t^{\alpha}, \\
u^{k}(x, t) & =u^{\text {initial }}(x, t)+\sum_{i=2}^{k} \frac{f_{i}(x)}{\Gamma(i \alpha+1)} t^{i \alpha}, \\
k & =2,3, \ldots,
\end{aligned}
$$

$$
\operatorname{Res}(x, t)=D_{t}^{2 \alpha} u(x, t)+G(x, t),
$$

where $G(x, t)=-\delta D_{x}^{6 \rho} u(x, t)-\beta D_{x}^{4 \sigma} u(x, t)-\gamma D_{x}^{2 \tau} u(x, t)-$ $\theta D_{x}^{2 \lambda} u^{2}(x, t)+4 \theta u^{2}(x, t)$,

$$
\begin{aligned}
\operatorname{Res}^{k}(x, t)= & D_{t}^{2 \alpha} u^{k}(x, t)+G^{k}(x, t), \\
G^{k}(x, t)= & -\delta D_{x}^{6 \rho} u^{k}(x, t)-\beta D_{x}^{4 \sigma} u^{k}(x, t) \\
& -\gamma D_{x}^{2 \tau} u^{k}(x, t)-\theta D_{x}^{2 \lambda}\left(u^{k}(x, t)\right)^{2} \\
& +4 \theta\left(u^{k}(x, t)\right)^{2}, \quad k=2,3, \ldots
\end{aligned}
$$

By (23) and (24), it is shown that

$$
\begin{gathered}
f_{i}(x)=-\left.D_{t}^{(i-2) \alpha} G^{i}(x, t)\right|_{t=0} \\
=\left.\delta D_{t}^{(i-2) \alpha} D_{x}^{6 \rho} u^{i}(x, t)\right|_{t=0} \\
+\left.\beta D_{t}^{(i-2) \alpha} D_{x}^{4 \sigma} u^{i}(x, t)\right|_{t=0} \\
+\left.\gamma D_{t}^{(i-2) \alpha} D_{x}^{2 \tau} u^{i}(x, t)\right|_{t=0}
\end{gathered}
$$

$$
\begin{aligned}
& +\left.\theta D_{t}^{(i-2) \alpha} D_{x}^{2 \lambda}\left(u^{i}(x, t)\right)^{2}\right|_{t=0} \\
& -\left.4 \theta D_{t}^{(i-2) \alpha}\left(u^{i}(x, t)\right)^{2}\right|_{t=0}=\delta D_{x}^{6 \rho} a_{i-2}(x) \\
& +\beta D_{x}^{4 \sigma} a_{i-2}(x)+\gamma D_{x}^{2 \tau} a_{i-2}(x) \\
& +\theta D_{x}^{2 \lambda} \Gamma((i-2) \alpha+1) \\
& \cdot \sum_{j=0}^{i-2} \frac{a_{j}(x) a_{i-2-j}(x)}{\Gamma(j \alpha+1) \Gamma((i-2-j) \alpha+1)} \\
& -4 \theta \Gamma((i-2) \alpha+1) \\
& \cdot \sum_{j=0}^{i-2} \frac{a_{j}(x) a_{i-2-j}(x)}{\Gamma(j \alpha+1) \Gamma((i-2-j) \alpha+1)} \triangleq a_{i}(x), \\
& \quad i=2,3,4, \ldots .
\end{aligned}
$$

So the $k$ th approximate solution of (41) is

$$
u^{k}(x, t)=\sum_{i=0}^{k} \frac{a_{i}(x)}{\Gamma(i \alpha+1)} t^{i \alpha}, \quad k=0,1,2,3, \ldots,
$$

where $a_{i}(x)(i=0,1,2,3, \ldots)$ can be found from (41) and (46).

Particularly, when $m=2, \beta=1, \gamma=1, \theta=1, \rho=1$, $\sigma=1, \tau=1, \lambda=1, a_{0}(x)=e^{x}$, and $a_{1}(x)=\sqrt{3} e^{x}$, the exact solution of (41) is

$$
u(x, t)=e^{x} E_{\alpha}\left(\sqrt{3} t^{\alpha}\right) .
$$

According to RPSM, we can obtain

$$
a_{i}(x)=(\sqrt{3})^{i} e^{x}, \quad i=0,1,2,3, \ldots
$$

And the $k$ th approximate solution of (41) is

$$
\begin{array}{r}
u^{k}(x, t)=e^{x} \sum_{i=0}^{k} \frac{a_{i}(x)}{\Gamma(i \alpha+1)} t^{i \alpha}=e^{x} \sum_{i=0}^{k} \frac{(\sqrt{3})^{i}}{\Gamma(i \alpha+1)} t^{i \alpha}, \\
k=0,1,2,3, \ldots .
\end{array}
$$

It is easy to see that

$$
\begin{aligned}
u(x, t) & =\lim _{k \rightarrow \infty} u^{k}(x, t)=\lim _{k \rightarrow \infty} e^{x} \sum_{i=0}^{k} \frac{(\sqrt{3})^{i}}{\Gamma(i \alpha+1)} t^{i \alpha} \\
& =e^{x} \sum_{n=0}^{\infty} \frac{\left(\sqrt{3} t^{\alpha}\right)^{n}}{\Gamma(n+1)}=e^{x} E_{\alpha}\left(\sqrt{3} t^{\alpha}\right) .
\end{aligned}
$$

So the FPS solution of (41) is

$$
u(x, t)=e^{x} E_{\alpha}\left(\sqrt{3} t^{\alpha}\right),
$$

which is equal to (48).

When $\alpha=1$, the exact solution is $u(x, t)=e^{x+\sqrt{3} t}$, and we also plot the images (see Figures 7, 8, 9, 10, 11, and 12). And it is clear that when $k=9, u^{k}(x, t)$ is closely approximate to $u(x, t)$. 


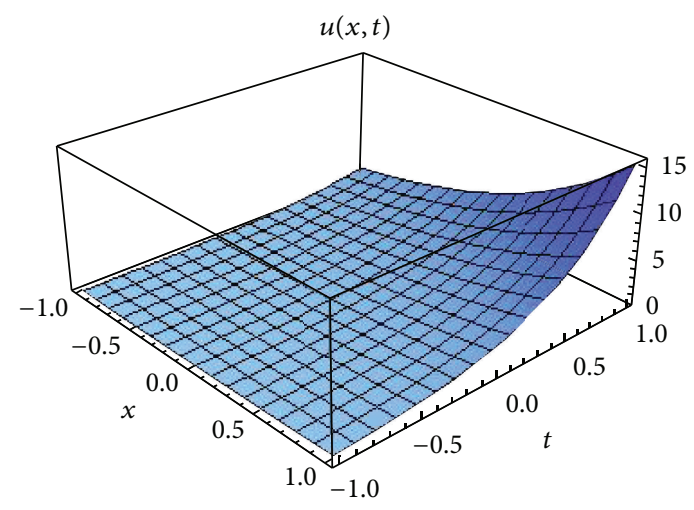

Figure 7: The image of $u(x, t)$.

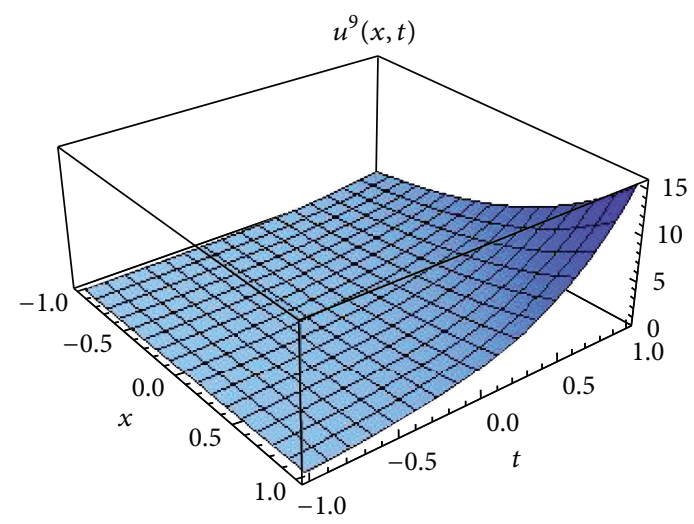

FIgURE 8: The image of $u^{9}(x, t)$.

5.3. 2nth-Order Time-Space Fractional Boussinesq Equations in $\mathbb{R}$. Let us consider the sixth-order time-space fractional Boussinesq equation:

$$
\begin{aligned}
D_{t}^{2 \alpha} u(x, t)= & \sum_{p=1}^{n} \beta_{p} D_{x}^{2 p \tau_{p}} u(x, t)+\theta D_{x}^{2} u^{2}(x, t) \\
& -4 \theta u^{2}(x, t) \\
u(x, 0)= & a_{0}(x) \\
\left.D_{t}^{\alpha} u(x, t)\right|_{t=0}= & a_{1}(x)
\end{aligned}
$$

where $u(x, t)$ is a field function, $(x, t) \in \mathbb{R} \times \mathbb{R}, 1 / 2<\alpha \leq$ $1,(2 p-1) / 2 p<\tau_{p} \leqslant 1, \beta_{p}$ are constant coefficients, $p=$ $1,2, \ldots, n$, and $a_{0}(x)$ and $a_{1}(x) \in C^{\infty}(\mathbb{R})$.

According to (25), the solution of (53) can be written in the following form:

$$
\begin{aligned}
& u(x, t)=\sum_{i=0}^{\infty} u_{i}(x, t) \\
& u_{i}(x, t)=C_{i}(x) t^{i \alpha}=\frac{f_{i}(x)}{\Gamma(i \alpha+1)} t^{i \alpha}
\end{aligned}
$$

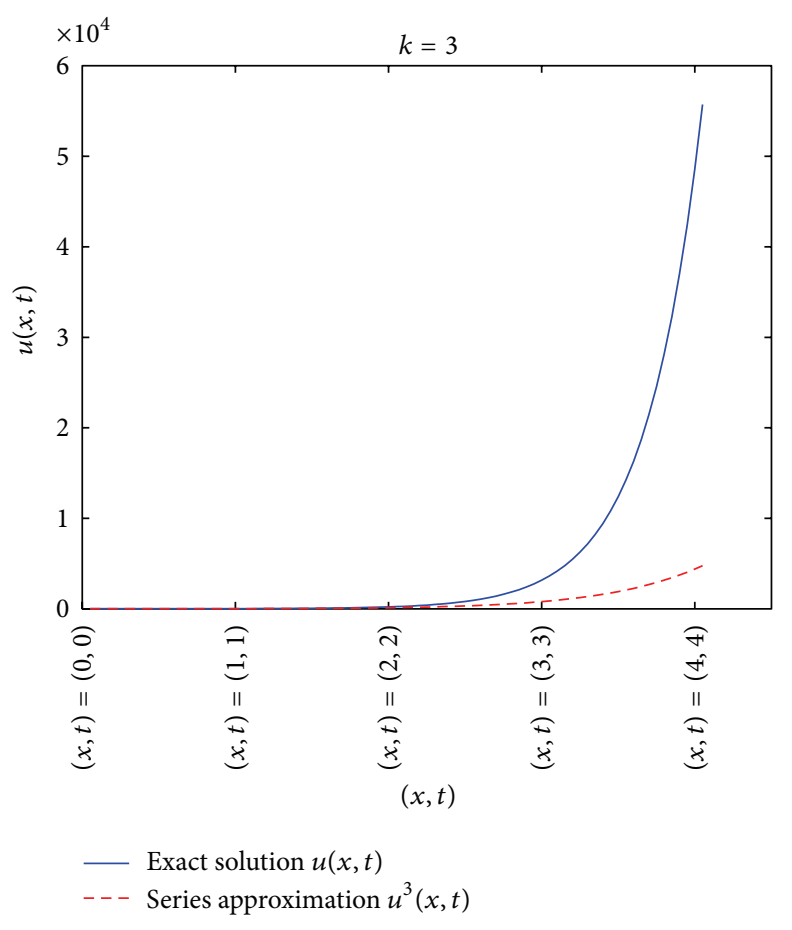

FIGURE 9: The image of $u(x, t)$ and $u^{3}(x, t)$.

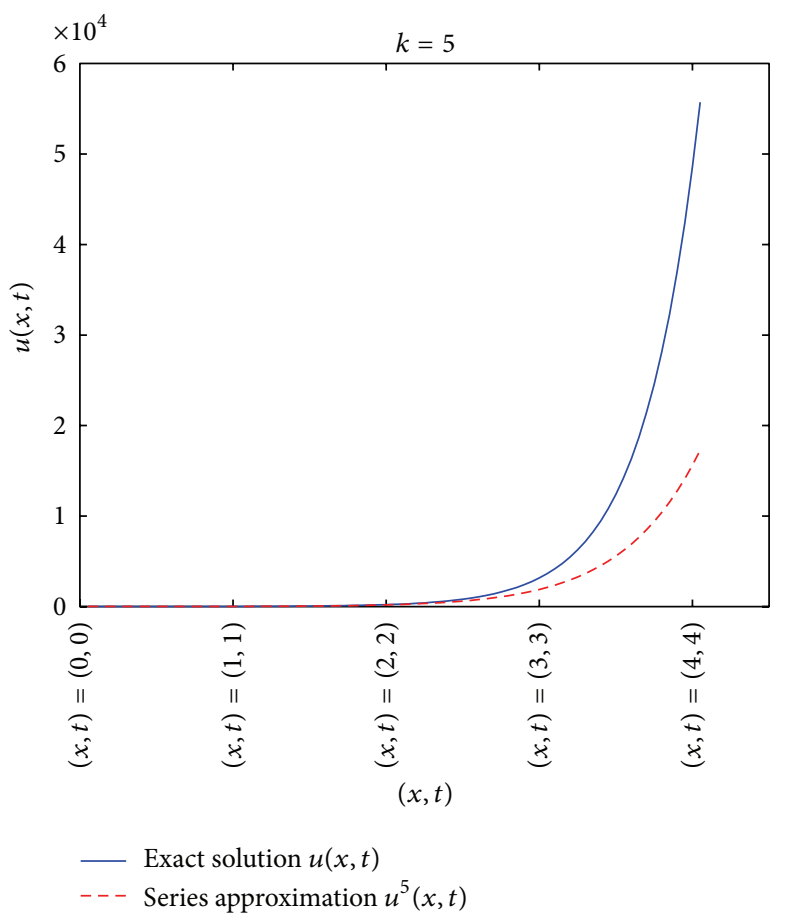

FIgURE 10: The image of $u(x, t)$ and $u^{5}(x, t)$.

Apparently, according to (17), we have

$$
\begin{aligned}
& f_{0}(x)=a_{0}(x), \\
& f_{1}(x)=a_{1}(x) .
\end{aligned}
$$




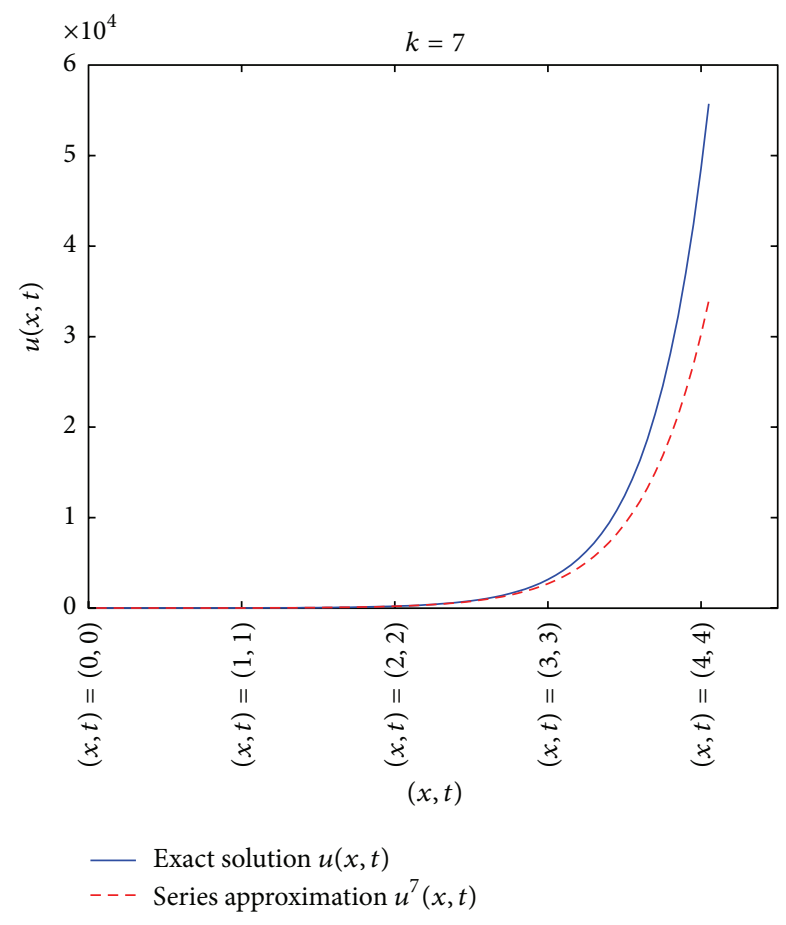

FIGURE 11: The image of $u(x, t)$ and $u^{7}(x, t)$.

By (18)-(22), we can obtain

$$
\begin{aligned}
u^{\text {initial }}(x, t) & =a_{0}(x)+\frac{a_{1}(x)}{\Gamma(\alpha+1)} t^{\alpha}, \\
u^{k}(x, t) & =u^{\text {initial }}(x, t)+\sum_{i=2}^{k} \frac{f_{i}(x)}{\Gamma(i \alpha+1)} t^{i \alpha}, \\
& (k=2,3, \ldots),
\end{aligned}
$$

$$
\operatorname{Res}(x, t)=D_{t}^{2 \alpha} u(x, t)+G(x, t)
$$

where $G(x, t)=-\sum_{p=1}^{n} \beta_{p} D_{x}^{2 p \tau_{p}} u(x, t)-\theta D_{x}^{2} u^{2}(x, t)+$ $4 \theta u^{2}(x, t)$

$$
\begin{aligned}
\operatorname{Res}^{k}(x, t)= & D_{t}^{2 \alpha} u^{k}(x, t)+G^{k}(x, t), \\
G^{k}(x, t)= & -\sum_{p=1}^{n} \beta_{p} D_{x}^{2 p \tau_{p}} u^{k}(x, t)-\theta D_{x}^{2}\left(u^{k}(x, t)\right)^{2} \\
& +4 \theta\left(u^{k}(x, t)\right)^{2}, \quad k=2,3, \ldots
\end{aligned}
$$

Using (23) and (24), it is shown that

$$
\begin{aligned}
& f_{i}(x)=-\left.D_{t}^{(i-2) \alpha} G^{i}(x, t)\right|_{t=0} \\
& =\left.D_{t}^{(i-2) \alpha}\left(\sum_{p=1}^{n} \beta_{p} D_{x}^{2 p \tau_{p}} u^{i}(x, t)\right)\right|_{t=0} \\
& \quad+\left.\theta D_{t}^{(i-2) \alpha}\left(u^{i}(x, t)\right)^{2}\right|_{t=0}
\end{aligned}
$$

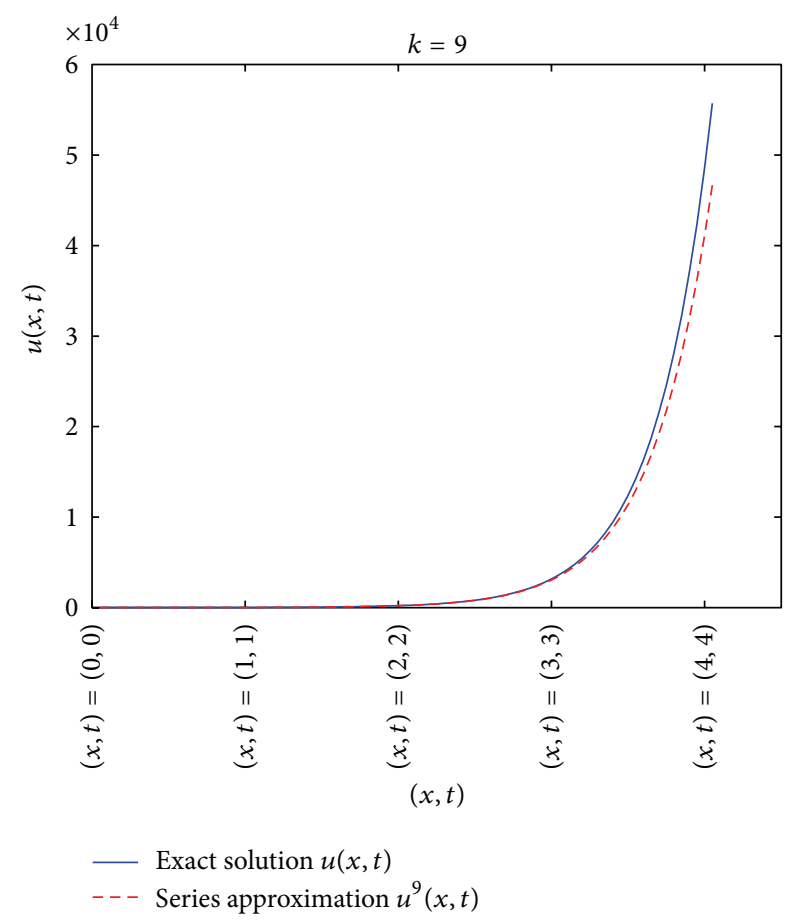

FIgURE 12: The image of $u(x, t)$ and $u^{9}(x, t)$.

$$
\begin{aligned}
& -\left.4 \theta D_{t}^{(i-2) \alpha}\left(u^{i}(x, t)\right)^{2}\right|_{t=0}=\sum_{p=1}^{n} \beta_{p} D_{x}^{2 p \tau_{p}} a_{i-2}(x) \\
& +\theta D_{x}^{2} \Gamma((i-2) \alpha+1) \\
& \cdot \sum_{j=0}^{i-2} \frac{a_{j}(x) a_{i-2-j}(x)}{\Gamma(j \alpha+1) \Gamma((i-2-j) \alpha+1)} \\
& -4 \theta \Gamma((i-2) \alpha+1) \\
& \cdot \sum_{j=0}^{i-2} \frac{a_{j}(x) a_{i-2-j}(x)}{\Gamma(j \alpha+1) \Gamma((i-2-j) \alpha+1)} \triangleq a_{i}(x), \\
& \quad i=2,3,4, \ldots
\end{aligned}
$$

So the $k$ th approximate solution of (53) is

$$
u^{k}(x, t)=\sum_{i=0}^{k} \frac{a_{i}(x)}{\Gamma(i \alpha+1)} t^{i \alpha}, \quad k=0,1,2,3, \ldots,
$$

where $a_{i}(x)(i=0,1,2,3, \ldots)$ can be found from (53) and (58).

When $m=2, \beta=1, \gamma=1, \theta=1, \tau_{p}=1, p=1,2, \ldots, n$, $\lambda=1, a_{0}(x)=e^{x}$, and $a_{1}(x)=\sqrt{n} e^{x}$, the exact solution of (53) is

$$
u(x, t)=e^{x} E_{\alpha}\left(\sqrt{n} t^{\alpha}\right) .
$$

According to RPSM, we have

$$
a_{i}(x)=(\sqrt{n})^{i} e^{x}, \quad i=0,1,2,3, \ldots
$$


And the $k$ th approximate solution of (53) is

$$
\begin{array}{r}
u^{k}(x, t)=e^{x} \sum_{i=0}^{k} \frac{a_{i}(x)}{\Gamma(i \alpha+1)} t^{i \alpha}=e^{x} \sum_{i=0}^{k} \frac{(\sqrt{n})^{i}}{\Gamma(i \alpha+1)} t^{i \alpha}, \\
k=0,1,2,3, \ldots .
\end{array}
$$

It is easy to see that

$$
\begin{aligned}
u(x, t) & =\lim _{k \rightarrow \infty} u^{k}(x, t)=\lim _{k \rightarrow \infty} e^{x} \sum_{i=0}^{k} \frac{(\sqrt{n})^{i}}{\Gamma(i \alpha+1)} t^{i \alpha} \\
& =e^{x} \sum_{n=0}^{\infty} \frac{\left(\sqrt{n} t^{\alpha}\right)^{n}}{\Gamma(n \alpha+1)}=e^{x} E_{\alpha}\left(\sqrt{n} t^{\alpha}\right) .
\end{aligned}
$$

So the FPS solution of (53) is

$$
u(x, t)=e^{x} E_{\alpha}\left(\sqrt{n} t^{\alpha}\right)
$$

which is equal to (60).

5.4. Fourth-Order Time-Space Fractional Boussinesq Equations in $\mathbb{R}^{2}$. Consider the fourth-order time-space fractional Boussinesq equation with two-dimensional space variables:

$$
\begin{aligned}
D_{t}^{2 \alpha} u(x, t)= & \beta_{1} D_{x_{1}}^{4 \sigma_{1}} u(x, t)+\beta_{2} D_{x_{2}}^{4 \sigma_{2}} u(x, t) \\
& +\gamma_{1} D_{x_{1}}^{2 \tau_{1}} u(x, t)+\gamma_{2} D_{x_{2}}^{2 \tau_{2}} u(x, t) \\
& +\theta_{1} D_{x_{1}}^{2 \lambda_{1}} u^{2}(x, t) \\
& +\theta_{2} D_{x_{2}}^{2 \lambda_{2}} u^{2}(x, t)-4 \theta_{1} u^{2}(x, t) \\
& -4 \theta_{2} u^{2}(x, t), \\
u(x, 0)= & a_{0}(x), \\
\left.D_{t}^{\alpha} u(x, t)\right|_{t=0}= & a_{1}(x),
\end{aligned}
$$

where $u(x, t)$ is a field function, $x=\left(x_{1}, x_{2}\right) \in \mathbb{R}^{2}, t \in \mathbb{R}$, $1 / 2<\alpha \leq 1,3 / 4<\sigma_{i} \leqslant 1,1 / 2<\tau_{i} \leqslant 1,1 / 2<\lambda_{i} \leqslant 1, \beta_{i}, \gamma_{i}$, $\theta_{i}(i=1,2)$ are constants, and $a_{0}(x)$ and $a_{1}(x) \in C^{\infty}\left(\mathbb{R}^{2}\right)$.

Before solving this problem, we give several notations for convenience:

$$
\begin{aligned}
& x=\left(x_{1}, x_{2}\right), \\
& \bar{x}:=x_{1}+x_{2}, \\
& \boldsymbol{\beta}=\left(\beta_{1}, \beta_{2}\right), \\
& \boldsymbol{\gamma}=\left(\gamma_{1}, \gamma_{2}\right), \\
& \boldsymbol{\theta}=\left(\theta_{1}, \theta_{2}\right), \\
& \boldsymbol{\lambda}=\left(\lambda_{1}, \lambda_{2}\right), \\
& \bar{\theta}:=\theta_{1}+\theta_{2},
\end{aligned}
$$

$$
\begin{aligned}
D_{x}^{j \omega}(\cdot) & :=\left(D_{x_{1}}^{j \omega_{1}}, D_{x_{2}}^{j \omega_{2}}\right)^{T}(\cdot), \\
\qquad \boldsymbol{\omega}=\left(\omega_{1}, \omega_{2}\right), j=2,4, & \\
\lambda D_{x}^{j}(\cdot): & \left(\lambda_{1}, \lambda_{2}\right)\left(D_{x_{1}}^{j}, D_{x_{2}}^{j \omega}\right)^{T}(\cdot) \\
& =\lambda_{1} D_{x_{1}}^{j \omega_{1}}(\cdot)+\lambda_{2} D_{x_{2}}^{j \omega_{2}}(\cdot) .
\end{aligned}
$$

So system (65) is equal to

$$
\begin{aligned}
D_{t}^{\alpha} u(x, t)= & \beta D_{x}^{4 \sigma} u(x, t)+\gamma D_{x}^{2 \tau} u(x, t) \\
& +\theta D_{x}^{2 \lambda} u^{2}(x, t)-4 \bar{\theta} u^{2}(x, t), \\
u(x, 0)= & a_{0}(x), \\
\left.D_{t}^{\alpha} u(x, t)\right|_{t=0}= & a_{1}(x) .
\end{aligned}
$$

According to (25), the solution of (67) can be written in the following form:

$$
\begin{aligned}
& u(x, t)=\sum_{n=0}^{\infty} u_{n}(x, t), \\
& u_{n}(x, t)=C_{n}(x) t^{n \alpha}=\frac{f_{n}(x)}{\Gamma(n \alpha+1)} t^{n \alpha}, \\
& n=0,1,2, \ldots
\end{aligned}
$$

Apparently, according to (17), we have

$$
\begin{aligned}
& f_{0}(x)=a_{0}(x), \\
& f_{1}(x)=a_{1}(x) .
\end{aligned}
$$

Using (18)-(22), we can obtain

$$
\begin{aligned}
u^{\text {initial }}(x, t) & =a_{0}(x)+\frac{a_{1}(x)}{\Gamma(\alpha+1)} t^{\alpha}, \\
u^{k}(x, t) & =u^{\text {initial }}(x, t)+\sum_{i=2}^{k} \frac{f_{i}(x)}{\Gamma(i \alpha+1)} t^{i \alpha}, \\
k & =2,3, \ldots,
\end{aligned}
$$

$$
\operatorname{Res}(x, t)=D_{t}^{2 \alpha} u(x, t)+G(x, t),
$$

where $G(x, t)=-\beta D_{x}^{4 \sigma} u(x, t)-\gamma D_{x}^{2 \tau} u(x, t)-\theta D_{x}^{2 \lambda} u^{2}(x, t)+$ $4 \bar{\theta} u^{2}(x, t)$,

$$
\begin{aligned}
\operatorname{Res}^{k}(x, t)= & D_{t}^{2 \alpha} u^{k}(x, t)+G^{k}(x, t), \\
G^{k}(x, t)= & -\beta D_{x}^{4 \sigma} u^{k}(x, t)-\gamma D_{x}^{2 \tau} u^{k}(x, t) \\
& -\boldsymbol{\theta} D_{x}^{2 \lambda}\left(u^{k}(x, t)\right)^{2}+4 \bar{\theta}\left(u^{k}(x, t)\right)^{2}, \\
& k=2,3, \ldots
\end{aligned}
$$


Using (23) and (24), it is shown that

$$
\begin{aligned}
f_{i}( & x)=-\left.D_{t}^{(i-2) \alpha} G^{i}(x, t)\right|_{t=0} \\
= & \left.\beta D_{t}^{(i-2) \alpha} D_{x}^{4 \sigma} u^{i}(x, t)\right|_{t=0} \\
& +\left.\gamma D_{t}^{(i-2) \alpha} D_{x}^{2 \tau} u^{i}(x, t)\right|_{t=0} \\
& +\left.\theta D_{t}^{(i-2) \alpha} D_{x}^{2 \lambda}\left(u^{i}(x, t)\right)^{2}\right|_{t=0} \\
& -\left.4 \bar{\theta} D_{t}^{(i-2) \alpha}\left(u^{i}(x, t)\right)^{2}\right|_{t=0}=\beta D_{x}^{4 \sigma} a_{i-2}(x) \\
& +\gamma D_{x}^{2 \tau} a_{i-2}(x)+\theta D_{x}^{2 \lambda} \Gamma((i-2) \alpha+1) \\
& \cdot \sum_{j=0}^{i-2} \frac{a_{j}(x) a_{i-2-j}(x)}{\Gamma(j \alpha+1) \Gamma((i-2-j) \alpha+1)} \\
& -4 \bar{\theta} \Gamma((i-2) \alpha+1) \\
& +\sum_{j=0}^{i-2} \frac{a_{j}(x) a_{i-2-j}(x)}{\Gamma(j \alpha+1) \Gamma((i-2-j) \alpha+1)} \triangleq a_{i}(x),
\end{aligned}
$$

So the $k$ th approximate solution of (67) is

$$
u^{k}(x, t)=\sum_{i=0}^{k} \frac{a_{i}(x)}{\Gamma(i \alpha+1)} t^{i \alpha}, \quad k=0,1,2,3, \ldots,
$$

where $a_{i}(x)(i=0,1,2,3, \ldots)$ can be found from (67) and (72).

Particularly when $m=2, \boldsymbol{\beta}=(1,1), \boldsymbol{\gamma}=(1,1), \boldsymbol{\theta}=(1,1)$, $\boldsymbol{\sigma}=(1,1), \boldsymbol{\tau}=(1,1), \boldsymbol{\lambda}=(1,1), a_{0}(x)=e^{\bar{x}}$, and $a_{1}(x)=$ $\sqrt{2} e^{\bar{x}}$, the exact solution of (67) is

$$
u(x, t)=e^{\bar{x}} E_{\alpha}\left(\sqrt{2} t^{\alpha}\right) .
$$

According to RPSM, we have

$$
a_{i}(x)=(\sqrt{2})^{i} e^{\bar{x}}, \quad i=0,1,2,3, \ldots
$$

And the $k$ th approximate solution of (67) is

$$
\begin{array}{r}
u^{k}(x, t)=e^{\bar{x}} \sum_{i=0}^{k} \frac{a_{i}(x)}{\Gamma(i \alpha+1)} t^{i \alpha}=e^{\bar{x}} \sum_{i=0}^{k} \frac{(\sqrt{2})^{i}}{\Gamma(i \alpha+1)} t^{i \alpha}, \\
k=0,1,2,3, \ldots .
\end{array}
$$

It is easy to see that

$$
\begin{aligned}
u(x, t) & =\lim _{k \rightarrow \infty} u^{k}(x, t)=\lim _{k \rightarrow \infty} e^{\bar{x}} \sum_{i=0}^{k} \frac{(\sqrt{2})^{i}}{\Gamma(i \alpha+1)} t^{i \alpha} \\
& =e^{\bar{x}} \sum_{n=0}^{\infty} \frac{\left(\sqrt{2} t^{\alpha}\right)^{n}}{\Gamma(n \alpha+1)}=e^{\bar{x}} E_{\alpha}\left(\sqrt{2} t^{\alpha}\right) .
\end{aligned}
$$

So the FPS solution of (67) is

$$
u(x, t)=e^{\bar{x}} E_{\alpha}\left(\sqrt{2} t^{\alpha}\right),
$$

which is equal to (74).
5.5. Fourth-Order Time-Space Fractional Boussinesq Equations in $\mathbb{R}^{n}$. Let us consider the fourth-order time-space fractional Boussinesq equation with $n$-dimensional space variables:

$$
\begin{aligned}
D_{t}^{2 \alpha} u(x, t)= & \sum_{p=1}^{n_{1}} \beta_{p} D_{x_{p}}^{4 \sigma_{p}} u(x, t)+\sum_{q=1}^{n_{2}} \gamma_{q} D_{x_{q}}^{2 \tau_{q}} u(x, t) \\
& +\sum_{r=1}^{n} \theta_{r} D_{x_{r}}^{2 \lambda_{r}} u^{2}(x, t) \\
& -\sum_{r=1}^{n} 4 \theta_{r} u^{2}(x, t), \\
u(x, 0)= & a_{0}(x), \\
\left.D_{t}^{\alpha} u(x, t)\right|_{t=0}= & a_{1}(x),
\end{aligned}
$$

where $u(x, t)$ is a field function, $x=\left(x_{1}, x_{2}, \ldots, x_{n}\right) \in \mathbb{R}^{n}$, $n \in \mathbb{N}, t \in \mathbb{R}, 1 / 2<\alpha \leq 1,3 / 4<\sigma_{p} \leqslant 1, p=1,2, \ldots, n_{1} \leq$ $n \in \mathbb{N}, 1 / 2<\tau_{q} \leqslant 1, q=1,2, \ldots, n_{2} \leq n \in \mathbb{N}, 1 / 2<\lambda_{r} \leqslant 1$, $r=1,2, \ldots, n, \beta_{p}\left(p=1,2, \ldots, n_{1}\right)$ and $\gamma_{q}\left(q=1,2, \ldots, n_{2}\right)$ are constants, and $a_{0}(x)$ and $a_{1}(x) \in C^{\infty}\left(\mathbb{R}^{n}\right)$.

For convenience, we give several notations:

$$
\begin{aligned}
& x=\left(x_{1}, x_{2}, \ldots, x_{n}\right), \\
& \bar{x}:=\sum_{i=1}^{n} x_{i}, \\
& \boldsymbol{\beta}=\left(\beta_{1}, \beta_{2}, \ldots, \beta_{n_{1}}\right), \\
& \boldsymbol{\gamma}=\left(\gamma_{1}, \gamma_{2}, \ldots, \gamma_{n_{2}}\right), \\
& \boldsymbol{\theta}=\left(\theta_{1}, \theta_{2}, \ldots, \theta_{n}\right), \\
& \boldsymbol{\lambda}=\left(\lambda_{1}, \lambda_{2}, \ldots, \lambda_{n}\right), \\
& \bar{\theta}=\sum_{i=1}^{n} \theta_{i},
\end{aligned}
$$

$$
\begin{aligned}
D_{x}^{j \omega}(\cdot): & =\left(D_{x_{1}}^{j \omega_{1}}, D_{x_{2}}^{j \omega_{2}}, \ldots, D_{x_{n}}^{j \omega_{n}}\right)^{T}(\cdot), \\
\boldsymbol{\omega} D_{x}^{j}(\cdot): & =\left(\omega_{1}, \omega_{2}, \ldots, \omega_{n}\right), j=2,4, \\
= & \sum_{i=1}^{n}\left(\lambda_{i}, \ldots, \lambda_{n}\right)\left(D_{x_{i}}^{j \omega_{i}}(\cdot)\right) .
\end{aligned}
$$

So system (79) is equal to

$$
\begin{aligned}
D_{t}^{2 \alpha} u(x, t)= & \beta D_{x}^{4 \sigma} u(x, t)+\gamma D_{x}^{2 \tau} u(x, t) \\
& +\theta D_{x}^{2 \lambda} u^{2}(x, t)-4 \bar{\theta} u^{2}(x, t), \\
u(x, 0)= & a_{0}(x), \\
\left.D_{t}^{\alpha} u(x, t)\right|_{t=0}= & a_{1}(x) .
\end{aligned}
$$


According to (25), the solution of (81) can be written in the following form:

$$
\begin{aligned}
& u(x, t)=\sum_{i=0}^{\infty} u_{i}(x, t), \\
& u_{i}(x, t)=C_{i}(x) t^{i \alpha}=\frac{f_{i}(x)}{\Gamma(i \alpha+1)} t^{i \alpha} \\
& \quad(i=0,1,2, \ldots) .
\end{aligned}
$$

Apparently, according to (17), we have

$$
\begin{aligned}
& f_{0}(x)=a_{0}(x), \\
& f_{1}(x)=a_{1}(x) .
\end{aligned}
$$

By (18)-(22), we can show that

$$
\begin{aligned}
u^{\text {initial }}(x, t) & =a_{0}(x)+\frac{a_{1}(x)}{\Gamma(\alpha+1)} t^{\alpha}, \\
u^{k}(x, t)=u^{\text {initial }}(x, t)+\sum_{i=2}^{k} \frac{f_{i}(x)}{\Gamma(i \alpha+1)} t^{i \alpha}, & (k=2,3, \ldots),
\end{aligned}
$$

$$
\operatorname{Res}(x, t)=D_{t}^{2 \alpha} u(x, t)+G(x, t)
$$

where $G(x, t)=-\beta D_{x}^{4 \sigma} u(x, t)-\gamma D_{x}^{2 \tau} u(x, t)-\theta D_{x}^{2 \lambda} u^{2}(x, t)+$ $4 \bar{\theta} u^{2}(x, t)$,

$$
\begin{aligned}
& \operatorname{Res}^{k}(x, t)= D_{t}^{2 \alpha} u^{k}(x, t)+G^{k}(x, t), \\
& G^{k}(x, t)=-\beta D_{x}^{4 \sigma} u^{k}(x, t)-\gamma D_{x}^{2 \tau} u^{k}(x, t) \\
&-\theta D_{x}^{2 \lambda}\left(u^{k}\right)^{2}(x, t)+4 \bar{\theta}\left(u^{k}\right)^{2}(x, t), \\
& k=2,3, \ldots
\end{aligned}
$$

According to (23) and (24), we can obtain

$$
\begin{aligned}
f_{i}(x) & =-\left.D_{t}^{(i-2) \alpha} G^{i}(x, t)\right|_{t=0} \\
= & \left.\beta D_{t}^{(i-2) \alpha} D_{x}^{4 \sigma} u^{i}(x, t)\right|_{t=0} \\
& +\left.\gamma D_{t}^{(i-2) \alpha} D_{x}^{2 \tau} u^{i}(x, t)\right|_{t=0} \\
& +\left.\theta D_{t}^{(i-2) \alpha} D_{x}^{2 \lambda}\left(u^{i}(x, t)\right)^{2}\right|_{t=0} \\
& -\left.4 \bar{\theta} D_{t}^{(i-2) \alpha}\left(u^{i}(x, t)\right)^{2}\right|_{t=0}=\beta D_{x}^{4 \sigma} a_{i-2}(x) \\
& +\gamma D_{x}^{2 \tau} a_{i-2}(x)+\theta D_{x}^{2 \lambda} \Gamma((i-2) \alpha+1) \\
& +\sum_{j=0}^{i-2} \frac{a_{j}(x) a_{i-2-j}(x)}{\Gamma(j \alpha+1) \Gamma((i-2-j) \alpha+1)} \\
& -4 \bar{\theta} \Gamma((i-2) \alpha+1)
\end{aligned}
$$

$$
\cdot \sum_{j=0}^{i-2} \frac{a_{j}(x) a_{i-2-j}(x)}{\Gamma(j \alpha+1) \Gamma((i-2-j) \alpha+1)} \triangleq a_{i}(x),
$$$$
i=2,3,4, \ldots
$$

So the $k$ th approximate solution of (81) is

$$
u^{k}(x, t)=\sum_{i=0}^{k} \frac{a_{i}(x)}{\Gamma(i \alpha+1)} t^{i \alpha}, \quad k=0,1,2,3, \ldots,
$$

where $a_{i}(x)(i=0,1,2,3, \ldots)$ can be found from (81) and (86).

When $m=2, \boldsymbol{\beta}=\underbrace{(1,1, \ldots, 1)}_{n}, \gamma=\underbrace{(1,1, \ldots, 1)}_{n}$, $\boldsymbol{\theta}=\underbrace{(1,1, \ldots, 1)}_{n}, \boldsymbol{\sigma}=\underbrace{(1,1, \ldots, 1)}_{n_{1}}, \boldsymbol{\tau}=\underbrace{(1,1, \ldots, 1)}_{n_{2}}, \boldsymbol{\lambda}=$ $\underbrace{(1,1, \ldots, 1)}_{n}, a_{0}(x)=e^{\bar{x}}$, and $a_{1}(x)=\sqrt{2} e^{\bar{x}}$. The exact solution of (81) is

$$
u(x, t)=e^{\bar{x}} E_{\alpha}\left(\sqrt{2} t^{\alpha}\right) .
$$

According to RPSM, we have

$$
a_{i}(x)=(\sqrt{2})^{i} e^{\bar{x}}, \quad i=0,1,2,3, \ldots
$$

And the $k$ th approximate solution of (81) is

$$
\begin{array}{r}
u^{k}(x, t)=e^{\bar{x}} \sum_{i=0}^{k} \frac{a_{i}(x)}{\Gamma(i \alpha+1)} t^{i \alpha}=e^{\bar{x}} \sum_{i=0}^{k} \frac{(\sqrt{2})^{i}}{\Gamma(i \alpha+1)} t^{i \alpha}, \\
k=0,1,2,3, \ldots
\end{array}
$$

It is easy to see that

$$
\begin{aligned}
u(x, t) & =\lim _{k \rightarrow \infty} u^{k}(x, t)=\lim _{k \rightarrow \infty} e^{\bar{x}} \sum_{i=0}^{k} \frac{(\sqrt{2})^{i}}{\Gamma(i \alpha+1)} t^{i \alpha} \\
& =e^{\bar{x}} \sum_{n=0}^{\infty} \frac{(\sqrt{2} t)^{n}}{\Gamma(n \alpha+1)}=e^{\bar{x}} E_{\alpha}\left(\sqrt{2} t^{\alpha}\right) .
\end{aligned}
$$

So the FPS solution of (81) is

$$
u(x, t)=e^{\bar{x}} E_{\alpha}\left(\sqrt{2} t^{\alpha}\right),
$$

which is equal to (88).

\section{Conclusion}

The RPSM method for constructing the fractional power series solutions for any-order time-space fractional Boussinesq equation with the initial values in $\mathbb{R}^{n}$ is presented in this paper. The present technique is performed, based mainly on generating a residual error function and then applying the generalized power series formula. The numerical simulation results reveal the validity and reliability of RPSM. That is to say, RPSM is extremely powerful in terms of constructing fractional power series solutions or approximate fractional power series solutions for any-order time-space fractional partial differential equations in scientific applications. 


\section{Appendix}

See Tables 1, 2, 3, and 4 .

\section{Competing Interests}

The authors declare that there are no competing interests regarding the publication of this paper.

\section{Acknowledgments}

The authors express their sincere thanks to Professor Yong Li for his instructions and many invaluable suggestions. This work is supported in part by NSFC Grants 11571065, 11401089, and 11271062 and Jilin Science and Technology Development Project.

\section{References}

[1] O. Abu Arqub, "Series solution of fuzzy differential equations under strongly generalized differentiability," Journal of Advanced Research in Applied Mathematics, vol. 5, no. 1, pp. 3152, 2013.

[2] J.-H. He, "Approximate analytical solution for seepage flow with fractional derivatives in porous media," Computer Methods in Applied Mechanics and Engineering, vol. 167, no. 1-2, pp. 57-68, 1998.

[3] G. W. Leibnitz, Letter from Hanover, Germany, September 30, 1695, to G. A L'Hospital, Leibnizen Mathematische Schriften, Olms, Hildesheim, Germany, 1849.

[4] A. Loverro, Fractional Calculus: History, Definitions and Applications for the Engineer, Department of Aerospace and Mechanical Engineering, University of Notre Dame, South Bend, Ind, USA, 2004.

[5] A. El-Ajou, O. Abu Arqub, and S. Momani, "Approximate analytical solution of the nonlinear fractional KdV-Burgers equation: a new iterative algorithm," Journal of Computational Physics, vol. 293, pp. 81-95, 2015.

[6] A. El-Ajou, O. Abu Arqub, Z. Al Zhour, and S. Momani, "New results on fractional power series: theories and applications," Entropy, vol. 15, no. 12, pp. 5305-5323, 2013.

[7] G. M. Bisci, V. D. Radulescu, and R. Servadei, Variational Methods for Nonlocal Fractional Problems, vol. 162, Cambridge University Press, 2016.

[8] V. Lakshmikantham, Theory of Fractional Dynamic Systems, Cambridge Scientific Publishers, 2009.

[9] K. S. Miller and B. Ross, An Introduction to the Fractional Calculus and Fractional Differential Equations, A Wiley-Interscience Publication, John Wiley \& Sons, New York, NY, USA, 1993.

[10] K. B. Oldham and J. Spanier, The Fractional Calculus, A Subsidiary of Harcourt Brace Jovanovich, Publishers, Academic Press, New York, NY, USA, 1974.

[11] I. Podlubny, Fractional Differential Equations, vol. 198 of Mathematics in Science and Engineering, Academic Press, San Diego, Calif, USA, 1999.

[12] S. G. Samko, A. A. Kilbas, and O. I. Marichev, Fractional Integrals and Derivatives, Gordon and Breach Science Publishers, Yverdon, Yverdon-les-Bains, Switzerland, 1993.

[13] B. J. West, M. Bologna, and P. Grigolini, Physics of Fractal Operators, Institute for Nonlinear Science, New York, NY, USA, 2003.
[14] O. Abu Arqub, Z. Abo-Hammour, R. Al-Badarneh, and S. Momani, "A reliable analytical method for solving higher-order initial value problems," Discrete Dynamics in Nature and Society, vol. 2013, Article ID 673829, 12 pages, 2013.

[15] O. A. Arqub, A. El-Ajou, and S. Momani, "Constructing and predicting solitary pattern solutions for nonlinear timefractional dispersive partial differential equations," Journal of Computational Physics, vol. 293, pp. 385-399, 2015.

[16] O. A. Arqub, A. El-Ajou, Z. Al Zhour, and S. Momani, "Multiple solutions of nonlinear boundary value problems of fractional order: a new analytic iterative technique," Entropy, vol. 16, no. 1, pp. 471-493, 2014.

[17] A. Sadighi and D. D. Ganji, "Solution of the generalized nonlinear boussinesq equation using homotopy perturbation and variational iteration methods," International Journal of Nonlinear Sciences and Numerical Simulation, vol. 8, no. 3, pp. 435-443, 2007.

[18] H. Taskesen, N. Polat, and A. Ertaş, "Global existence and decay of solutions for the generalized bad Boussinesq equation," Applied Mathematics, vol. 28, no. 3, pp. 253-268, 2013.

[19] J. Sabatier, O. P. Agrawal, and J. A. T. Machado, Eds., Advances in Fractional Calculus, Springer, Dordrecht, The Netherlands, 2007.

[20] A.-M. Wazwaz, Partial Differential Equations, CRC Press, New York, NY, USA, 2002.

[21] T. A. Abassy, M. A. El-Tawil, and H. El-Zoheiry, "Modified variational iteration method for Boussinesq equation," Computers \& Mathematics with Applications, vol. 54, no. 7-8, pp. 955-965, 2007.

[22] J. Choudhury, Numerical and asymptotic investigation of the stationary-propagating localized solutions of the Boussinesq equation in two dimensions [Ph.D. thesis], University of Louisiana at Lafayette, Lafayette, Lo, USA; ProQuest, Ann Arbor, Mich, USA, 2008.

[23] S. A. El-Wakil and E. M. Abulwafa, "Formulation and solution of space-time fractional Boussinesq equation," Nonlinear Dynamics, vol. 80, no. 1-2, pp. 167-175, 2015.

[24] F. Xu, Y. Gao, and W. Zhang, "Construction of analytic solution for time-fractional Boussinesq equation using iterative method," Advances in Mathematical Physics, vol. 2015, Article ID 506140, 7 pages, 2015.

[25] H. Jafari, N. Kadkhoda, and D. Baleanu, "Fractional Lie group method of the time-fractional Boussinesq equation," Nonlinear Dynamics, vol. 81, no. 3, pp. 1569-1574, 2015.

[26] H. Beyer and S. Kempfle, "Definition of physically consistent damping laws with fractional derivatives," Zeitschrift für Angewandte Mathematik und Mechanik, vol. 75, no. 8, pp. 623-635, 1995.

[27] A. A. Kilbas, H. M. Srivastava, and J. J. Trujillo, Theory and Applications of Fractional Differential Equations, vol. 204 of North-Holland Mathematics Studies, Elsevier, Amsterdam, The Netherlands, 2006.

[28] V. E. Tarasov, Fractional Dynamics: Applications of Fractional Calculus to Dynamics of Particles, Fields and Media, Springer Science \& Business Media, Berlin, Germany, 2011.

[29] V. V. Uchaikin, Fractional Derivatives for Physicists and Engineers: Nonlinear Physical Science, Nonlinear Physical Science, Springer, Berlin, Germany, 2013. 


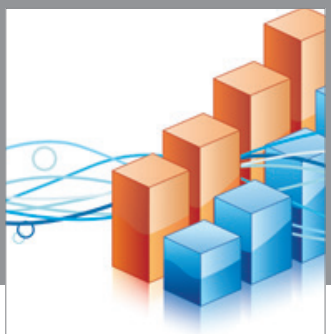

Advances in

Operations Research

vatem alat4

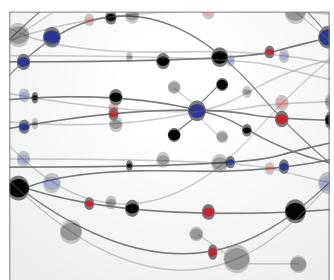

\section{The Scientific} World Journal
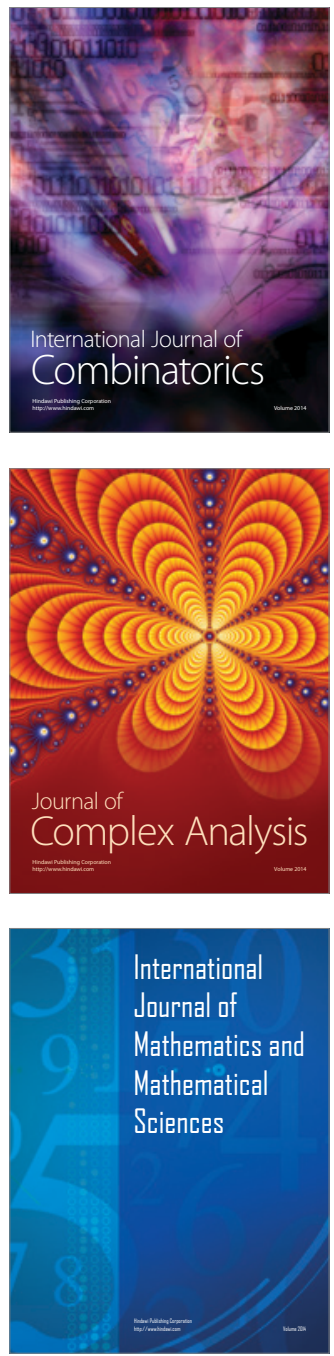
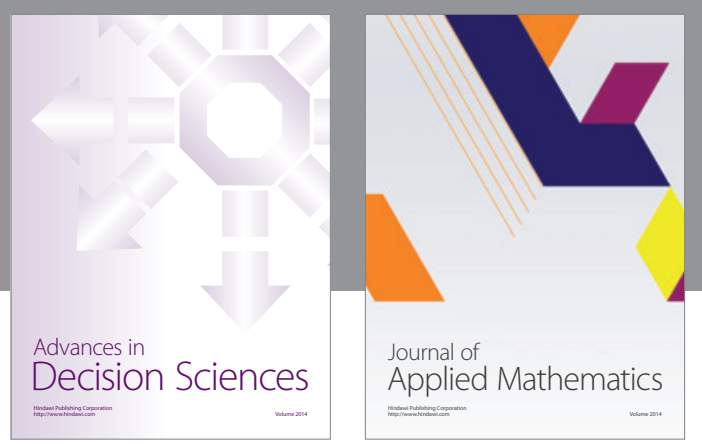

Algebra

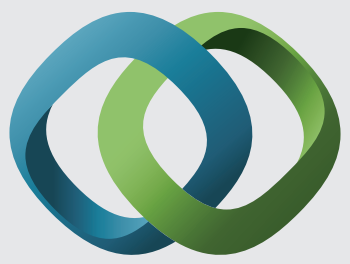

\section{Hindawi}

Submit your manuscripts at

http://www.hindawi.com
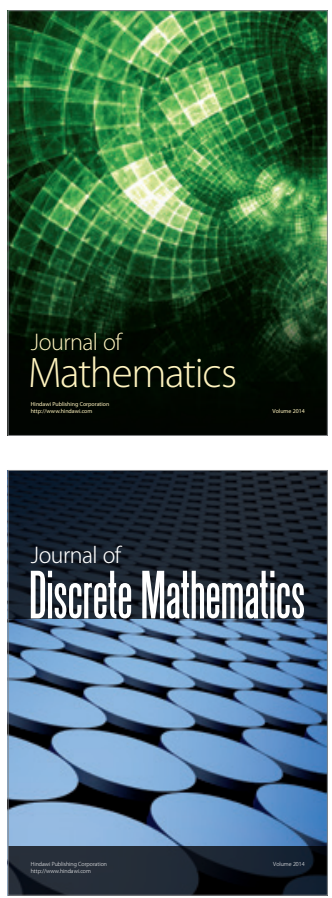

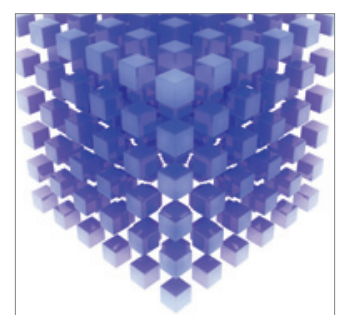

Mathematical Problems in Engineering
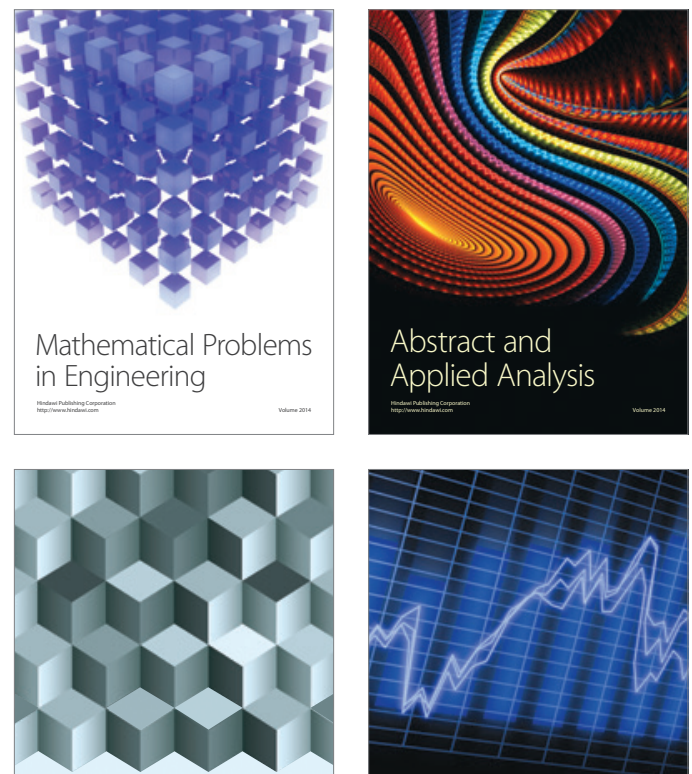

Journal of

Function Spaces

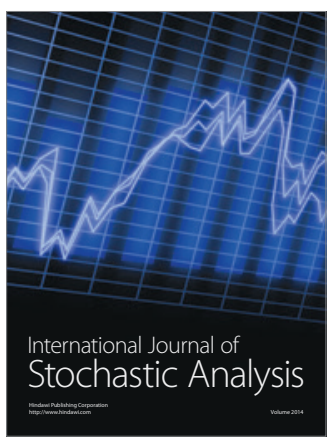

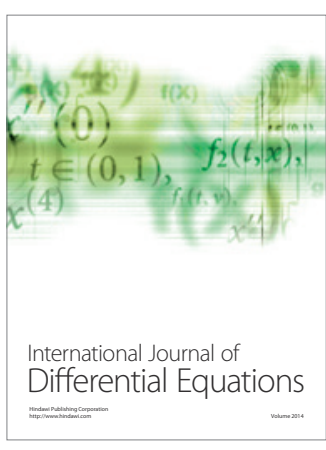
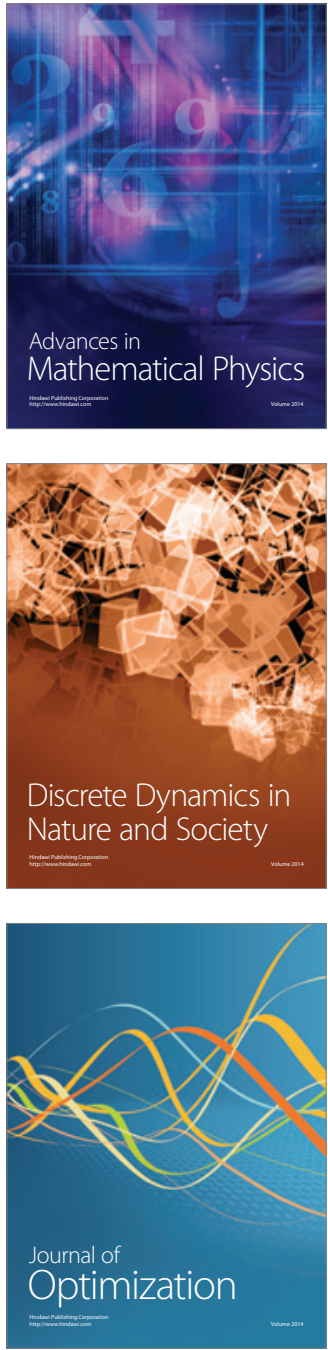